\title{
ROBUST AND SCALABLE H-ADAPTIVE AGGREGATED UNFITTED FINITE ELEMENTS FOR INTERFACE ELLIPTIC PROBLEMS
}

\author{
Eric Neiva ${ }^{\mathrm{a}, \mathrm{b}, *}$ and Santiago Badia ${ }^{\mathrm{c}, \mathrm{a}}$ \\ ${ }^{a}$ CIMNE - Centre Internacional de Mètodes Numèrics en Enginyeria, \\ Edifici C1, Campus Nord UPC, C. Gran Capità S/N, 08034 Barcelona, Spain. \\ ${ }^{\mathrm{b}}$ Department of Civil and Environmental Engineering, Universitat Politècnica de Catalunya, \\ Edifici C2, Campus Nord UPC, C. Jordi Girona 1-3, 08034 Barcelona, Spain. \\ ${ }^{\mathrm{c}}$ School of Mathematics, Monash University, Clayton, Victoria, 3800, Australia.
}

\begin{abstract}
This work introduces a novel, fully robust and highly-scalable, $h$-adaptive aggregated unfitted finite element method for large-scale interface elliptic problems. The new method is based on a recent distributed-memory implementation of the aggregated finite element method atop a highly-scalable Cartesian forest-of-trees mesh engine. It follows the classical approach of weakly coupling nonmatching discretisations at the interface to model internal discontinuities at the interface. We propose a natural extension of a single-domain parallel cell aggregation scheme to problems with a finite number of interfaces; it straightforwardly leads to aggregated finite element spaces that have the structure of a Cartesian product. We demonstrate, through standard numerical analysis and exhaustive numerical experimentation on several complex Poisson and linear elasticity benchmarks, that the new technique enjoys the following properties: well-posedness, robustness with respect to cut location and material contrast, optimal (h-adaptive) approximation properties, high scalability and easy implementation in large-scale finite element codes. As a result, the method offers great potential as a useful finite element solver for large-scale interface problems modelled by partial differential equations.
\end{abstract}

Keywords: Unfitted finite elements - Interface linear elasticity · Interface Poisson · Adaptive mesh refinement · High performance scientific computing

\section{INTRODUCTION}

Unfitted finite element (FE) methods are generating considerable interest in many practical situations. Their ability to handle complex geometries, avoiding cumbersome and time-consuming body-fitted mesh generation, makes them especially appealing for large-scale simulations. They have been successfully exploited in many applications with moving interfaces, such as fracture mechanics [1-3], fluid-structure interaction [4-7], twophase and free surface flows [8-10], and in applications with varying domains, such as shape or topology optimisation [11, 12], additive manufacturing [13, 14], and stochastic geometry problems [15]. In the numerical community, unfitted FE methods receive different denominations. When the motivation is to capture (moving) interfaces, they are usually referred to as eXtended FE methods (XFEM) [16]. On the other hand, when the goal is to simulate a problem using a (usually simple) background mesh, they are denoted as unfitted or embedded or immersed techniques; see, e.g. the cutFEM method [17], the cutIGA method [18], the immersed boundary method [19], the finite cell method [20], the shifted boundary method [21], the immersogeometric method [22] and discontinuous Galerkin (DG) methods with cell aggregation [23-27].

This work investigates unfitted FE methods in large scale simulations of interface problems modelled with partial differential equations (PDEs). Typical approaches pursued to model internal discontinuities across the unfitted interface are (1) weak coupling of nonmatching discretisations [28], (2) local partition-of-unity enrichments [29] and (3) Lagrange multiplier or mortar methods [30, 31], although all three are closely connected [32, 33]. This work focuses on the first approach. It broadly consists in dividing the mesh into two (sub)meshes that overlap in cut cells. It leads to FE approximations that have the structure of a Cartesian product. Transmission conditions on the unfitted interface are then weakly enforced by means of penalty [34] or Nitsche [35] formulations, among others.

In the context of unfitted interface methods, the main challenge is to derive robust methods for large material contrast across the interface. Indeed, naive variational formulations may exhibit poor stability in this regime, e.g. average numerical flux weighting in Nitsche methods produces inaccurate and oscillating approximation of interface quantities [36]. On the other hand, large material contrast problems are prone to the so-called small cut cell problem. This issue is formally circumscribed to the unfitted boundary case and it is associated with

\footnotetext{
*Corresponding author.

Emails: eneiva@cimne.upc.edu, santiago.badia@monash.edu

May 29, 2022
} 
cut cells with arbitrarily small intersection with the physical domain. Unless a specific technique mitigates the problem, numerical integration on these badly-cut cells leads to severe ill-conditioning problems [37, 38]. Since unfitted boundary problems can be interpreted as a limiting case of large contrast interface ones, the latter are not completely immune to the issue [39].

Despite vast literature on the topic [40-43], fewer authors achieve formulations that are fully robust and optimal, regardless of cut location and material contrast. A notable exception is the family of methods that rely on ghost penalty $[17,44]$. These works adopt approach (1) and enrich the variational formulation with suitable stabilization terms defined in the faces of cut cells; the resulting formulation is robust to cut location. Besides, robustness w.r.t. material contrast is achieved by using the so-called harmonic weights in the Nitsche formulation, a typical approach in body-fitted DG methods [45]. As a result, the condition number of the diagonally-scaled system matrix becomes independent of the material contrast [17, 39]. However, research in this area has tended to overlook scalability and $h p$-adaptivity, which are essential aspects in applications to large-scale problems. These aspects have been considered by the finite cell method community [46, 47], but robustness w.r.t. material contrast has barely received their attention.

Research over the past few years is turning to an alternative approach to ensure robustness with respect to cut location, the so-called cell aggregation or cell agglomeration techniques. This approach is very natural in DG methods, as they can be easily formulated on agglomerated meshes [40, 48, 49]. The extension of these ideas to conforming discretisations is less obvious, since such aggregation process requires to keep trace continuity among cells. With this aim, the (continuous Galerkin (CG)) aggregated unfitted finite element method (FEM), referred to as AgFEM [38], is grounded on a discrete extension operator from well-posed to ill-posed degrees of freedom (DOFs). This operator is defined in terms of a cell aggregation and is amenable to arbitrarily complex 3D geometries and $h$-adaptivity [50]. In spite of this, research has been restricted so far to unfitted boundary elliptic [38] or Stokes [51] problems. Aggregation has also been used for CG [52] methods, but the resulting scheme relies on the assumption that the aggregates can always be rectangles. However, such assumption is wrong, even in two-dimensions; aggregates have more complicated shapes in general geometries and meshes. The authors in [52] picked an elementary 2D circular Poisson problem in a square with a circular inclusion, discretised with a uniform Cartesian grid, in a mesh in which rectangular aggregates only where possible. Aggregation has been recently employed for hybrid-high order (HHO) [53], even though these methods are not considering face aggregation strategies and thus, their trace unknowns can lead to ill-posed problems.

The main goal of this work is to present a novel aggregated FE method for interface elliptic boundary value problems (BVPs). In contrast with other existing CG methods, we clearly show that interface AgFEM enjoys overall well-behaved numerical properties and remarkable large-scale capability. In particular, we demonstrate, with theoretical results and thorough numerical experimentation, well-posedness, robustness w.r.t. to cut location and material contrast, optimal ( $h$-adaptive) approximation properties, high scalability and ease of implementation in high-performance computing (HPC) FE codes. The paper gives full insight into AgFEM, as a large-scale FE solver for complex interface problems modelled by PDEs. It is also intended to provide guidance in exploiting other unfitted CG methods by aggregation for interface problems.

The outline of this work is as follows. We assume first an embedded (multiple) $n$-interface geometrical setting in Section 2.1. Next, we extend the single-domain cell aggregation method in [38] to $n$-interface problems, in Section 2.2. Cell aggregation can be carried out independently on each subdomain and reuse, with little effort, existing distributed-memory implementations of the single-domain algorithm [54]. In Section 2.3, we define AgFE spaces for embedded $n$-interfaces; we see that they easily accommodate the interface-overlapping mesh approach in [28]. Afterwards, we restrict ourselves to the approximation of single interface linear elasticity problems, see Section 3.1. We derive a similar formulation to body-fitted DG methods [55], using the symmetric interior penalty method and harmonic average weights, to weakly enforce interface conditions, see Section 3.2. Numerical analysis, proving well-posedness and a priori error estimates, are also covered there; all results are stable with respect to cut location and material contrast. We implement the method in the large-scale FE software package FEMPAR [56], which exploits the highly-scalable forest-of-tree mesh engine p4est [57] for $h$-adaptivity. In the numerical tests of Section 4 , we consider both the linear elasticity and Poisson equations as model problems on several complex geometries and several $h p$-FEM standard benchmarks. We numerically assess optimal convergence rates on uniform and $h$-adaptive meshes, robustness with respect to cut location and material contrast, and weak-scalability. Finally, we report the main conclusions and contributions of the work in Section 5. 


\section{The AGgregATED UNFITTED FINITE ELEMENT METHOD ON INTERFACE PROBLEMS}

2.1. Embedded interface geometry setup. Let $\Omega \subset \mathbb{R}^{d}$, with $d=2,3$ denoting the space dimension, be an open, bounded, connected domain, with Lipschitz boundary $\partial \Omega$. Since we seek to analyse problems with multiple physics and/or phases, let $\left\{\Omega^{i}\right\}_{i=1}^{N}$ be a partition of $\Omega$ into $N$ subdomains $\Omega^{i}$ with Lipschitz boundaries $\partial \Omega^{i}$. Let now $\Gamma_{0} \doteq \bigcup_{i=1}^{N} \partial \Omega^{i} \backslash \partial \Omega$ denote the skeleton of the partition. Equivalently, there is a partition of $\Gamma_{0}$ into $\Gamma^{i j} \doteq \partial \Omega^{i} \cap \partial \Omega^{j}$, such that $\Gamma_{0} \doteq \bigcup_{i, j=1}^{N} \Gamma^{i j}$. Let $N_{0}$ denote the number of non-empty $\Gamma^{i j}$, for all $i, j=1, \ldots, N$. The setting is represented in Figure $1 \mathrm{~A}$.

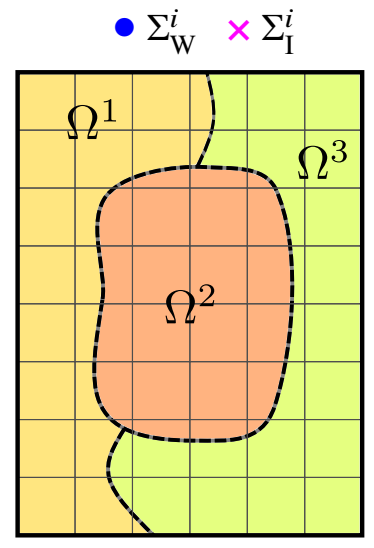

(A) $\Omega, \mathcal{T}_{h}$ and (dashed) $\Gamma_{0}$

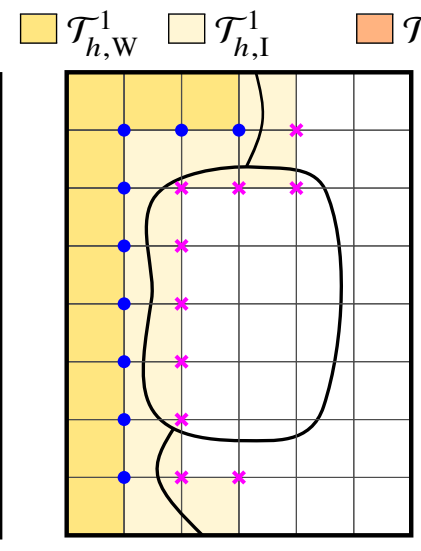

(в) $\mathcal{T}_{h, \mathrm{~A}}^{1}$ and $\mathcal{V}_{h, \mathrm{~A}}^{1}$

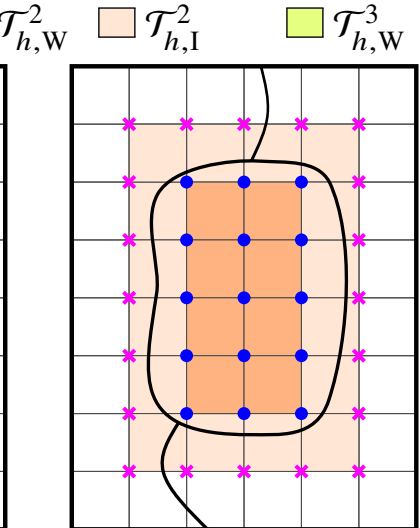

(c) $\mathcal{T}_{h, \mathrm{~A}}^{2}$ and $\mathcal{V}_{h, \mathrm{~A}}^{2}$

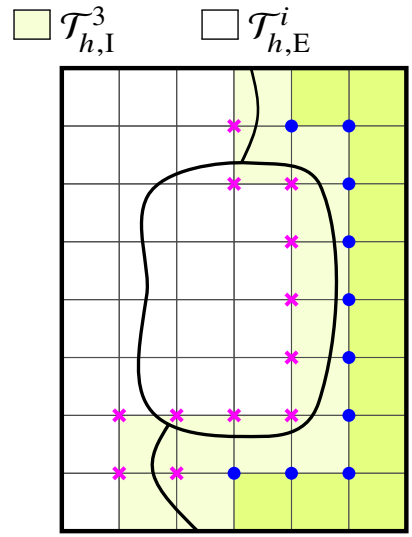

(D) $\mathcal{T}_{h, \mathrm{~A}}^{3}$ and $\mathcal{V}_{h, \mathrm{~A}}^{3}$

FIGURE 1. An embedded interface geometry setup for $N=3$ and $\eta_{0}=1$, i.e. well-posed if and only if interior and ill-posed if and only if cut. The boundary of the physical domain $\partial \Omega$ conforms to the mesh $\mathcal{T}_{h}$, whereas the skeleton $\Gamma_{0}$ is immersed in it. $\left\{\mathcal{T}_{h, \mathrm{~A}}^{i}\right\}_{i=1}^{3}$ forms a partition of $\mathcal{T}_{h}$, overlapping at cells cut by the skeleton $\Gamma_{0}$. As a result, degrees of freedom on cut cells are doubled or tripled (assuming linear lagrangian FEs). We consider partitions of DOFs in $\mathcal{V}_{h, \mathrm{~A}}^{i}$ into well-posed $\Sigma_{\mathrm{W}}^{i}$ and ill-posed $\Sigma_{\mathrm{I}}^{i}$ DOFs (note that we omit Dirichlet DOFs). Ill-posed DOFs are constrained in terms of well-posed DOFs, see Equation (1) and Figure 3.

We introduce now a typical embedded interface setup. To focus on the interface problem, we assume that $\Omega$ can be easily meshed with, e.g. Cartesian grids or unstructured $d$-simplexes, such that the external boundary $\partial \Omega$ conforms to the mesh, whereas $\Gamma_{0}$ remains immersed, as shown in Figure $1 \mathrm{~A}$. For simplicity in the exposition, let us consider that the mesh is body-fitted with respect to $\partial \Omega$, even though the general case can readily be tackled using the techniques in [38]. Instead, in this article, we focus on the extension of these techniques to resolve immersed interfaces. According to this, let $\mathcal{T}_{h}$ be a partition of $\Omega$ into cells, the so-called background mesh. Any $T \in \mathcal{T}_{h}$ is the image of a differentiable homeomorphism $\Phi_{T}$ over a set of admissible open reference $d$-polytopes [56], such as $d$-simplexes or $d$-cubes. We let $\mathcal{T}_{h}$ be non-conforming, i.e. there can be hanging vertices, edges or faces. We assume that the mesh is shape-regular and $h_{T}$ represents the characteristic size of the cell $T \in \mathcal{T}_{h}$.

We assume, without loss of generality, that the immersed skeleton $\Gamma_{0}$ is represented by the zero level-set of one or several known scalar functions, the so-called level-set functions, or by other means, e.g. from 3D CAD data, using techniques to compute the intersection between cell edges and surfaces (see, e.g. [58]). We also assume that we have suitable techniques (e.g. for local integration) to deal with cells that are intersected by more than one interface $\Gamma^{i j}$. For any cell $T \in \mathcal{T}_{h}$, we define the quantity

$$
\eta_{T}^{i} \doteq \frac{\operatorname{meas}_{d}\left(T \cap \Omega^{i}\right)}{\operatorname{meas}_{d}(T)}, \quad \eta_{T} \in[0,1], \quad i=1, \ldots, N,
$$

and a user-defined parameter $\eta_{0} \in(0,1]$, referred to as the well-posedness threshold. To isolate badly cut cells, we classify cells of $\mathcal{T}_{h}$ in terms of $\eta_{T}^{i}$ and $\eta_{0}$; it leads to subsets of $\mathcal{T}_{h}$ of the form

$$
\mathcal{T}_{h, \mathrm{~W}}^{i}=\left\{T \in \mathcal{T}_{h}: \eta_{T}^{i} \geq \eta_{0}\right\}, \quad \mathcal{T}_{h, \mathrm{I}}^{i}=\left\{T \in \mathcal{T}_{h}: \eta_{0}>\eta_{T}^{i}>0\right\}, \quad \mathcal{T}_{h, \mathrm{E}}^{i}=\left\{T \in \mathcal{T}_{h}: \eta_{T}^{i}=0\right\},
$$

for $i=1, \ldots, N . \mathcal{T}_{h, \mathrm{~W}}^{i}, \mathcal{T}_{h, \mathrm{I}}^{i}$ and $\mathcal{T}_{h, \mathrm{E}}^{i}$ are the well-posed (W), ill-posed (I) and exterior cells (E) associated with subdomain $\Omega^{i} . \mathcal{T}_{h, \mathrm{~W}}^{i}$ contains interior cells or those with a large portion inside $\Omega^{i}, \mathcal{T}_{h, \mathrm{I}}^{i}$, those with small cut portions in $\Omega^{i}$, and $\mathcal{T}_{h, \mathrm{E}}^{i}$ those with empty intersection with $\Omega^{i}$. We remark that, for $\eta_{0}=1$, well- or ill-posed cells coincide with interior or cut cells. By definition, each triplet $\left\{\mathcal{T}_{h, \mathrm{~W}}^{i}, \mathcal{T}_{h, \mathrm{I}}^{i}, \mathcal{T}_{h, \mathrm{E}}^{i}\right\}, i=1, \ldots, N$, forms a nonoverlapping partition of $\mathcal{T}_{h}$. We denote the union of cells of $\mathcal{T}_{h, \mathrm{~W}}^{i}, \mathcal{T}_{h, \mathrm{I}}^{i}$ and $\mathcal{T}_{h, \mathrm{E}}^{i}$ by $\Omega_{\mathrm{W}}^{i}, \Omega_{\mathrm{I}}^{i}$ and 
$\Omega_{\mathrm{E}}^{i}$, e.g. $\Omega_{\mathrm{W}}^{i}=\bigcup_{T \in \mathcal{T}_{h, \mathrm{~W}}^{i}} \bar{T}$. We also introduce the active meshes and domains, given by $\mathcal{T}_{h, \mathrm{~A}}^{i} \doteq \mathcal{T}_{h, \mathrm{~W}}^{i} \cup \mathcal{T}_{h, \mathrm{I}}^{i}$ and $\Omega_{\mathrm{A}}^{i} \doteq \Omega_{\mathrm{W}}^{i} \cup \Omega_{\mathrm{I}}^{i}, i=1, \ldots, N$; note that $\Omega^{i} \subset \Omega_{\mathrm{A}}^{i}$. It follows that $\left\{\mathcal{T}_{h, \mathrm{~A}}^{i}\right\}_{i=1}^{N}$ is a partition of $\mathcal{T}_{h}$, overlapping at cells cut by the skeleton $\Gamma_{0}$, see Figures $1 \mathrm{~B}-1 \mathrm{C}-1 \mathrm{D}$. We observe that our geometrical configuration generalises to multiple interfaces the classical approach adopted in, e.g. [17, 28], for single interface problems. Indeed, for $N=2\left(N_{0}=1\right),\left\{\mathcal{T}_{h, \mathrm{~A}}^{i}\right\}_{i=1}^{2}$ is an overlapping partition of $\mathcal{T}_{h}$, that divides the mesh into two (sub)meshes, where cells cut by the interface are doubled.

2.2. Cell aggregation with multiple interfaces. Cell aggregation for single-domain problems is well-covered in previous works, e.g. [38]; here, we limit ourselves to lay out the extension of the rationale to problems posed in domains with multiple interfaces, introduced in Section 2.1. We recall that aggregated FE spaces are grounded on a map, the so-called root cell map. This map associates any ill-posed cell with a well-posed cell, by means of a cell aggregation scheme, described in, e.g. [50, Algorithm 2.2].

In our context, we assume we carry out cell aggregation independently on each active mesh $\mathcal{T}_{h, \mathrm{~A}}^{i}, i=1, \ldots, N$, as illustrated in Figure 2; it yields the $i$-th root cell maps $\mathcal{R}^{i}: \mathcal{T}_{h, \mathrm{~A}}^{i} \rightarrow \mathcal{T}_{h, \mathrm{~W}}^{i}$. For any $T \in \mathcal{T}_{h, \mathrm{~W}}^{i}$, we refer to $A_{T}^{i} \doteq\left(\mathcal{R}^{i}\right)^{-1}(T)$ as a cell aggregate rooted at $T$. By construction of $\mathcal{R}^{i}$, aggregates take the form $A_{T}^{i}=\left\{T_{j}\right\}_{0 \leq j \leq m_{T}}$, where $T_{0}=T \in \mathcal{T}_{h, \mathrm{~W}}^{i}$ and $T_{j} \in \mathcal{T}_{h, \mathrm{I}}^{i}, 1 \leq j \leq m_{T}$, i.e. they are composed of several ill-posed cells and a unique (root) well-posed cell. Furthermore, aggregates are connected; they are also disjoint in $\mathcal{T}_{h, \mathrm{~A}}^{i}$, i.e. for any $T, T^{\prime} \in \mathcal{T}_{h, \mathrm{~A}}^{i}$, we have that $A_{\mathcal{R}^{i}(T)} \cap A_{\mathcal{R}^{i}\left(T^{\prime}\right)}=\emptyset$ or $\mathcal{R}^{i}(T) \equiv \mathcal{R}^{i}\left(T^{\prime}\right)$. It follows that $\mathcal{T}_{h, \mathrm{ag}}^{i} \doteq\left\{A_{T}^{i}\right\}_{T \in \mathcal{T}_{h, \mathrm{~W}}^{i}}$ are partitions of $\mathcal{T}_{h, \mathrm{~A}}^{i}$ into cell aggregates, for all $i=1, \ldots, N$. We observe that cell aggregation schemes only use the local information of each $\mathcal{T}_{h, \mathrm{~A}}^{i}, i=1, \ldots, N$; there is no coupling between active (sub)meshes. As a result, implementation of a multiple-domain cell aggregation scheme can fully reuse a single-domain counterpart.

$\mathcal{T}_{h, \mathrm{ag}}^{1}: \square$ aggregated $\square$ not aggregated $\mathcal{T}_{h, \mathrm{ag}}^{2}: \square$ agg. $\square$ not agg. $\quad \mathcal{T}_{h, \mathrm{ag}}^{3}: \square$ agg. $\square$ not agg. $\square \mathcal{T}_{h, \mathrm{E}}^{i}$

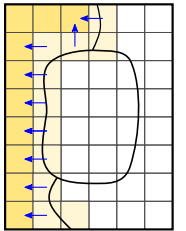

(A) Step 1

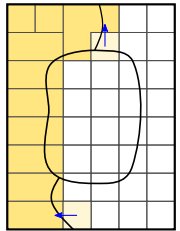

(в) Step 2

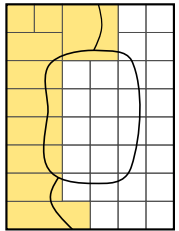

(c) Step 3

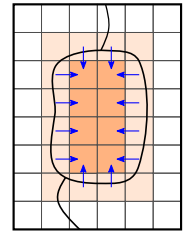

(D) Step 1

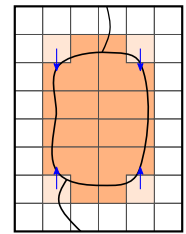

(E) Step 2

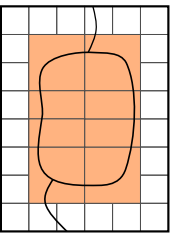

(F) Step 3

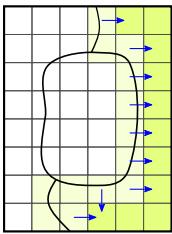

(G) Step 1

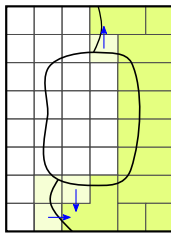

(н) Step 2

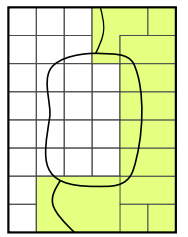

(I) Step 3

FIgURE 2. Cell aggregation on the three active meshes $\mathcal{T}_{h, \mathrm{~A}}^{i}, i=1,2,3$, of Figure 1 . The algorithm is detailed in, e.g. [50, Algorithm 2.2]. First, it marks well-posed cells as individual aggregates (Step 1). Then, aggregates grow iteratively, by attaching adjacent ill-posed cells to them (Step 2). The procedure stops when $\mathcal{T}_{h, \mathrm{~A}}^{i}, i=1,2,3$ is covered by aggregates (Step 3). This operation gives the root cell map $\mathcal{R}^{i}, i=1,2,3$. We observe that the scheme runs independently on each mesh with the local information provided by $\mathcal{T}_{h, \mathrm{~A}}^{i}, i=1,2,3$. Hence, implementation can reuse a single-domain cell aggregation scheme.

2.3. Aggregated Lagrangian finite element spaces. As stated in Section 1, we consider the common approach $[17,28,36]$ of building FE spaces on top of interface-overlapping meshes; it leads to FE approximations that have a Cartesian product structure. In our case, we aim to construct a CG AgFE space on top of the aggregated overlapping mesh $\left\{\mathcal{T}_{h \text {,ag }}^{i}\right\}_{i=1}^{N}$. We will see that we can straightforwardly exploit the single-domain methodology in [38] to derive an AgFE space on each aggregated mesh $\mathcal{T}_{h \text {,ag }}^{i}$ and, from here, a global FE space in $\mathcal{T}_{h}$ of the form $\mathcal{V}_{h, \text { ag }}^{1} \times \ldots \times \mathcal{V}_{h, \text { ag }}^{N}$.

For the sake of simplicity, we assume that the PDE problem posed in $\Omega$ is such that there is a single scalarvalued field associated to each subdomain $\Omega^{i}$. We also assume discretisations with Lagrangian FEs. In any case, the exposition can be generalised to other FEs, e.g. Nédélec [59], vector/tensor fields and multiple fields per $\Omega^{i}$. We also consider same cell topology everywhere in $\mathcal{T}_{h}$ and $\mathcal{T}_{h}$ conforming; although AgFE spaces on top of nonconforming meshes are fully covered in [50] and numerical tests in Section 4 run on Cartesian tree-based (nonconforming) meshes [60]. Lastly, we omit treatment of strong Dirichlet boundary conditions in the discussion below, although they can be easily taken care of, using standard approaches.

We denote by $\mathcal{V}(T)$ a vector space of functions defined on $T \in \mathcal{T}_{h}$. For $d$-simplex meshes, we define the local space $\mathcal{V}(T) \doteq \mathcal{P}_{q}(T)$, i.e. the space of polynomials of order less or equal to $q$ in the variables $x_{1}, \ldots, x_{d}$. For $d$-cubes, we define $\mathcal{V}(T) \doteq Q_{q}(T)$, i.e. the space of polynomials that are of degree less or equal to $q$ with respect to each variable in $x_{1}, \ldots, x_{d}$. In the numerical examples, we limit ourselves to rectangular or 
hexahedral cells and linear or quadratic shape functions, i.e. $\mathcal{V}(T) \doteq Q_{1}(T)$ or $\mathcal{V}(T) \doteq Q_{2}(T)$. To simplify notation, we define the elemental functional spaces $\mathcal{V}(T)$ in the physical cell $T \subset \Omega$ (even though our computer implementation relies on reference parametric spaces, as usual). Since we take on Lagrangian FEs, the basis for $\mathcal{V}(T)$ is the Lagrangian basis (of order $q$ ) on $T$; we assume same order everywhere in $\mathcal{T}_{h}$. We denote by $\Sigma_{T}$ the set of Lagrangian nodes of order $q$ of cell $T$, i.e. the set of local DOFs in $T$. There is a one-to-one mapping between nodes $\sigma \in \Sigma_{T}$ and shape functions $\phi_{T}^{\sigma}(\boldsymbol{x})$ such that $\phi_{T}^{\sigma}\left(\boldsymbol{x}^{\sigma^{\prime}}\right)=\delta_{\sigma \sigma^{\prime}}$, where $\boldsymbol{x}^{\sigma^{\prime}}$ are the space coordinates of node $\sigma^{\prime}$ and $\delta$ is the Kronecker delta.

Since we seek a global aggregated FE space of the form $\mathcal{V}_{h \text {,ag }}^{1} \times \ldots \times \mathcal{V}_{h \text {,ag }}^{N}$, we start by defining the subdomain members $\mathcal{V}_{h, \mathrm{gg}}^{i}, i=1, \ldots, N$. Thus, all notation and definitions in the next paragraphs are subdomain-local, i.e. referred to any subdomain $\Omega^{i} \subset \Omega, i=1, \ldots, N$, unless stated otherwise. According to this, let $\Sigma_{\mathrm{A}}^{i}$ refer to the set of (subdomain-)active DOFs of $\mathcal{T}_{h, \mathrm{~A}}^{i}$. We introduce next a local-to-subdomain DOF map $\sigma^{i}\left(T, \sigma^{\prime}\right) \in \Sigma_{\mathrm{A}}^{i}$, with $\sigma^{\prime} \in \Sigma_{T}$ and $T \in \mathcal{T}_{h}$. In CG methods, $\sigma^{i}$ is obtained by gluing together DOFs located in the same geometrical position; this operation leads to $\mathrm{C}^{0}$-continuous approximations. With this notation, we can define a standard FE space in $\mathcal{T}_{h, \mathrm{~A}}^{i}$ of the form

$$
\mathcal{V}_{h, \mathrm{~A}}^{i} \doteq\left\{v^{i} \in C^{0}\left(\Omega_{\mathrm{A}}^{i}\right):\left.v^{i}\right|_{T} \in \mathcal{V}(T), \forall T \in \mathcal{T}_{h, \mathrm{~A}}^{i}\right\} .
$$

It is well-known that, when the discrete FE problem is only integrated in $\Omega^{i}$, direct usage of $\mathcal{V}_{h, \mathrm{~A}}^{i}$ leads to arbitrarily ill-conditioned linear systems [37]. To solve this issue, we resort to the aggregated FEM [38, 51]. The main idea is to remove from $\mathcal{V}_{h, \mathrm{~A}}^{i}$ problematic DOFs, associated with small cut cells, by constraining them as a linear combination of DOFs with local support in a (well-posed) cell of $\mathcal{T}_{h, \mathrm{~W}}^{i}$. It leads to the aggregated subspace of $\mathcal{V}_{h, \mathrm{~A}}^{i}$, namely $\mathcal{V}_{h \text {,ag }}^{i}$, that gets rid of the aforementioned ill-conditioning issues.

In order to define $\mathcal{V}_{h \text {, ag }}^{i}$, the key is to realise that our context is analogous to one considering a single-domain unfitted-boundary problem, taking $\Omega^{i}$ as the physical domain embedded in $\Omega$. The former case is extensively covered in [38]. Hence, we can follow the same steps to derive $\mathcal{V}_{h \text {,ag }}^{i}$. According to this, let us define the set of well-posed DOFs as $\Sigma_{\mathrm{W}}^{i} \doteq \bigcup_{T \in \mathcal{T}_{h, \mathrm{~W}}^{i}} \Sigma_{T}$ and the set of ill-posed DOFs as $\Sigma_{\mathrm{I}}^{i} \doteq \Sigma_{\mathrm{A}}^{i} \backslash \Sigma_{\mathrm{W}}^{i}$, see Figure 1. Obviously, $\left\{\Sigma_{\mathrm{W}}^{i}, \Sigma_{\mathrm{I}}^{i}\right\}$ forms a partition of $\Sigma_{\mathrm{A}}^{i}$. $\Sigma_{\mathrm{W}}^{i}$ gathers all DOFs that have local support in (well-posed) cells of $\mathcal{T}_{h, \mathrm{~W}}^{i}$, while $\Sigma_{\mathrm{I}}^{i}$ isolates all DOFs, that potentially have arbitrarily small compact support and must be constrained in terms of well-posed DOFs of $\Sigma_{\mathrm{W}}^{i}$.

To compute ill-posed DOF constraints, we proceed as usual in AgFE methods. First, we compose the root cell map $\mathcal{R}^{i}: \mathcal{T}_{h, \mathrm{~A}}^{i} \rightarrow \mathcal{T}_{h, \mathrm{~W}}^{i}$ of Section 2.2, with a map between ill-posed DOFs $\Sigma_{\mathrm{I}}^{i}$ and ill-posed cells $\mathcal{T}_{h, \mathrm{I}}^{i}$ Specifically, we assign each ill-posed DOF to one of its surrounding ill-posed cells. The chosen cell is then mapped onto a well-posed cell via $\mathcal{R}^{i}$. Thus, the outcome of this composition is a map $\mathcal{K}^{i}: \Sigma_{\mathrm{I}}^{i} \rightarrow \mathcal{T}_{h, \mathrm{~W}}^{i}$, that assigns an ill-posed DOF to a well-posed cell via cell aggregation; see formal definitions in, e.g. [38, 54]. Following this, given $v^{i} \in \mathcal{V}_{h, \mathrm{~A}}^{i}$ and $\sigma \in \Sigma_{\mathrm{I}}^{i}$, we linearly extrapolate the nodal value of $\sigma$, namely $v_{\sigma}^{i} \in \mathbb{R}$, with the values at the local DOFs of its root cell $\mathcal{K}^{i}(\sigma)$. It leads to the constraint (see Figure 3 )

$$
v_{\sigma}^{i}=\sum_{\sigma^{\prime} \in \Sigma_{\mathcal{K}^{i}(\sigma)}} C_{\sigma \sigma^{\prime}} v_{\sigma^{\prime}}^{i}, \quad \text { with } C_{\sigma \sigma^{\prime}} \doteq \phi_{\mathcal{K}^{i}(\sigma)}^{\sigma^{\prime}}\left(\boldsymbol{x}^{\sigma}\right)
$$

As a result, the AgFE space can be readily defined as

$$
\mathcal{V}_{h, \mathrm{ag}}^{i} \doteq\left\{v^{i} \in \mathcal{V}_{h, \mathrm{~A}}^{i}: v_{\sigma}^{i}=\sum_{\sigma^{\prime} \in \Sigma_{\mathcal{K}^{i}(\sigma)}} C_{\sigma \sigma^{\prime}} v_{\sigma^{\prime}}^{i}, \forall \sigma \in \Sigma_{\mathrm{I}}^{i}\right\} .
$$

It is clear that $\mathcal{V}_{h, \text { ag }}^{i} \subset \mathcal{V}_{h, \mathrm{~A}}^{i}$. Further details, such as the form of (subdomain-wise) shape functions of $\mathcal{V}_{h, \mathrm{~A}}^{i}$, are not covered here, as they are analogous to those in [38].

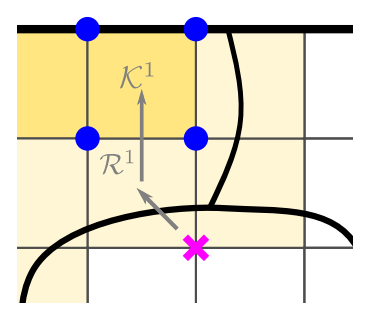

Figure 3. Close-up of Figure 1B illustrating an ill-posed DOF $(\times)$ in $\mathcal{T}_{h, \mathrm{~A}}^{1}$ mapped to a well-posed cell via $\mathcal{K}^{1}$. The resulting constraining DOFs, i.e. $\Sigma_{\mathcal{K}^{i}}$, are marked with $\bullet$ 
After defining independent AgFE spaces in $\Omega^{i}, i=1, \ldots, N$, a global aggregated FE space $\mathcal{V}_{h, \text { ag }}$ is straightforwardly derived as the Cartesian product of subdomain counterparts, i.e. $\mathcal{V}_{h, \text { ag }} \doteq \mathcal{V}_{h \text {,ag }}^{1} \times \ldots \times \mathcal{V}_{h \text {,ag }}^{N}$. We remark that, as $\mathcal{T}_{h, \mathrm{~A}}^{i}$ overlaps in cells cutting the skeleton $\Gamma_{0}$, DOFs lying on a cut cell are mapped to as many different global DOFs, as active meshes overlapping the cell, via the local-to-subdomain DOF map $\sigma^{i}$. However, some replicated DOFs may be marked as ill-posed and become constrained. As a result, they do not increase the size of the linear system. ${ }^{1}$

\section{APPROXIMATION OF UNFITTED INTERFACE ELLIPTIC PROBLEMS}

In this section, we address the approximation of compressible linear elasticity problems with the AgFEM. Extension of the method below to truly incompressible materials can be carried out with the mixed AgFEM in [51]. We introduce first the continuous interface problem (2) and prove that the weak formulation (5)-(6) is well-posed. Afterwards, we consider a consistent Nitsche's method (7) to discretise the problem with AgFEM. We conclude by examining well-posedness and approximability properties of the discrete problem (7), which lead to optimal a priori error estimates independent of the cut configuration.

From this point onwards, we restrict ourselves to single interface problems with two subdomains, i.e. there is a unique physical interface $\Gamma_{0} \equiv \Gamma^{12}$; henceforth denoted simply by $\Gamma$. This assumption contributes to conciseness and readability; all concepts presented here can be easily extended to the general case with an arbitrary number of subdomains. For the sake of the numerical analysis, let $\Gamma$ be a smooth manifold with bounded curvature. To distinguish the two subdomains, we use superscripts,+- instead of 1,2 , e.g. the subdomains are denoted by $\Omega^{+}$ and $\Omega^{-}$. In addition, we employ superscript $\alpha \in\{+,-\}$ to refer to any of the subdomains and \pm to refer to the broken domain, i.e. $\Omega^{ \pm} \doteq \Omega^{+} \cup \Omega^{-}$.

Before describing the model problem and approximation, we introduce some additional notation. Let $\boldsymbol{v}$ be a smooth enough vector or tensor function defined in $\Omega$. We denote by $\left.v^{\alpha} \doteq v\right|_{\Omega^{\alpha}}$ the restriction of $v$ into $\Omega^{\alpha}$; conversely, given $v^{\alpha}$ defined in $\Omega^{\alpha}$, we identify the pair $\left\{v^{+}, v^{-}\right\}$with the function $v$ in $\Omega^{ \pm}$, that is equal to $v^{\alpha}$ in $\Omega^{\alpha}$. On the interface, we define $\left.\boldsymbol{v}^{+}\right|_{\Gamma}(x)=\lim _{\epsilon \rightarrow 0^{+}} \boldsymbol{v}\left(\boldsymbol{x}-\epsilon \boldsymbol{n}^{+}\right)$and $\left.\boldsymbol{v}^{-}\right|_{\Gamma}(\boldsymbol{x})=\lim _{\epsilon \rightarrow 0^{-}} \boldsymbol{v}\left(\boldsymbol{x}+\epsilon \boldsymbol{n}^{-}\right)$, where $\boldsymbol{n}^{\alpha}$ is the outward pointing normal to $\Omega^{\alpha}$. We define the jump of $\boldsymbol{v}$ across $\Gamma$ by $\left.\llbracket v \rrbracket \doteq v^{+}\right|_{\Gamma}-\left.v^{-}\right|_{\Gamma}$ and the weighted average of $\boldsymbol{v}$ on $\Gamma$ as $\left.\{\{\boldsymbol{v}\}\} \doteq w^{+} \boldsymbol{v}^{+}\right|_{\Gamma}+\left.w^{-} \boldsymbol{v}^{-}\right|_{\Gamma}$, with $0 \leq w^{\alpha} \leq 1$ and $w^{+}+w^{-}=1$.

On the other hand, we use standard notation for Sobolev spaces (see, e.g. [61]). For instance, the $L^{2}(\omega)$ norm is denoted by $\|\cdot\|_{L^{2}(\omega)}$, the $H^{1}(\omega)$ norm as $\|\cdot\|_{H^{1}(\omega)}$ and the $H^{1}(\omega)$ seminorm as $|\cdot|_{H^{1}(\omega)}$. Given the two disjoint open connected subdomains $\Omega^{+}, \Omega^{-} \subset \mathbb{R}^{d}$, the Sobolev spaces of the form $H^{s}\left(\Omega^{+}\right) \times H^{s}\left(\Omega^{-}\right)$ are represented with $H^{s}\left(\Omega^{ \pm}\right)$, endowed with the norm $\|\cdot\|_{H^{s}\left(\Omega^{ \pm}\right)} \doteq\left(\|\cdot\|_{H^{s}\left(\Omega^{+}\right)}^{2}+\|\cdot\|_{H^{s}\left(\Omega^{-}\right)}^{2}\right)^{1 / 2}$; analogously for seminorms. Vector-valued Sobolev spaces are represented with boldface letters. We use common notation $A \lesssim B$ or $A \gtrsim B$ to denote that $A \leq C B$ or $A \geq C B$ for some positive constant $C$. In this work, constants may depend on the order of the FE space, the shape and size of $\Omega$ and $\Gamma$, and the user-defined value $\eta_{0}$, but they may not depend on the mesh-interface intersection (i.e. how the cells are intersected), the mesh size of the background mesh, or the contrast of the physical parameters at both sides of the interface.

Moreover, let us assume that the aggregate size is bounded by a constant times $h_{T}$, where $T$ is the root of the aggregate. This can be shown to hold when assuming that the ratio between the size of two neighbouring cells cannot be arbitrarily large, e.g. using standard 2:1 balance in adaptive non-conforming tree meshes or a patch-local quasi-regularity assumption on unstructured meshes (see also [38, Lemma 2.2]).

Lastly, we introduce the set of faces $\mathcal{F}_{h}$ that are generated after the intersection of $\Gamma$ and the mesh $\mathcal{T}_{h}$, i.e. $\mathcal{F}_{h} \doteq\left\{\bigcup_{T \in \mathcal{T}_{h}} \Gamma \cap T\right\} \cup\left\{\bigcup_{T, T^{\prime} \in \mathcal{T}_{h}: T \neq T^{\prime}} \Gamma \cap\left(\bar{T} \cap \overline{T^{\prime}}\right)\right\}$; a face $F$ in $\mathcal{F}_{h}$ can be on the boundary of the background mesh cells or intersect the cells. In the subsequent analysis, there is no difference between the two cases and, thus, we do not distinguish among them. Given $F \in \mathcal{F}_{h}$, we let $T_{F}^{\alpha} \in \mathcal{T}_{h, \mathrm{~A}}^{\alpha}$ such that $F \cap \overline{\Omega^{\alpha}} \subset \overline{T_{F}^{\alpha}}$ and $h_{T_{F}} \doteq \max \left\{h_{T_{F}^{+}}, h_{T_{F}^{-}}\right\}$. Note that $T_{F}^{+} \equiv T_{F}^{-}$for faces that intersect the cells.

\footnotetext{
${ }^{1}$ In this sense, AgFEM departs from other unfitted techniques that rely on the same interface-overlapping mesh approach, such as cutFEM. In those cases, the problem is incremented by the number of replicated DOFs. In particular, the total number of (free) DOFs is $\sum_{i=1, N}\left|\Sigma_{\mathrm{A}}^{i}\right|$. In contrast, the size of the linear system in AgFEM is always smaller and bounded above by $\sum_{i=1, N}\left|\sum_{\mathrm{A}}^{i}\right|$; indeed, the total number of DOFs is regulated by the well-posedness threshold $\eta_{0}$. For $\eta_{0}$ equal to zero, we would exactly have $\sum_{i=1, N}\left|\Sigma_{\mathrm{A}}^{i}\right|$, but this is the standard XFEM case, which is useless because it does not get rid of the small cut cell problem. The larger $\eta_{0}$ is, the more cells are marked as ill-posed and thus the number of DOFs reduced, because more DOFs are constrained and do not appear in the (reduced) linear system. In the aggregation process, replicated DOFs on the interface cells are eliminated and one can easily end up with a problem even smaller than the original FE problem. In any case, the interface region usually demands more refined meshes due to small scale local effects. This is accomplished by combining AgFEM with adaptive mesh refinement and coarsening (see [50]).
} 
Our main goal is to prove that all constants being used in the analysis are independent of $h$ and the cellinterface intersection. They may depend, though, on the well-posedness threshold $\eta_{0}$, the shape and size of $\Omega$ and $\Gamma$, and the order of the FE approximation. The key strategy in the analysis, in order to prove robustness w.r.t. the small cut cell problem, is to build upon well-behaved properties, that enjoy AgFE spaces in BVPs posed on unfitted boundaries, i.e. where $\partial \Omega$ is unfitted, instead of $\Gamma$; these properties have been thoroughly covered in $[38,51]$. We will often refer to them, without repeating details, to keep the presentation short.

Besides, we also aim to gain some control on the robustness of method (7) to material contrast. Since we rule out incompressibility, we adopt the quotient of $\mu$ coefficients at either sides of $\Gamma$ as the measure of material contrast, i.e. we consider $\mu_{+} / \mu_{-}$in the numerical experiments. Therefore, we can follow the usual approach for the Laplacian problem, adopted in body-fitted DG [45] and small-cut-stable unfitted [17] methods. In particular, we employ the so-called harmonic average weights, that is $w_{+} \doteq \frac{\mu_{-}}{\mu_{+}+\mu_{-}}$and $w_{-} \doteq \frac{\mu_{+}}{\mu_{+}+\mu_{-}}$. Clearly, $w_{\alpha}, \alpha \in\{+,-\}$, does not depend on cut location, only on material contrast. We will denote the harmonic average of $\mu$ by $\bar{\mu} \doteq \frac{2 \mu_{+} \mu_{-}}{\mu_{+}+\mu_{-}}$. We have that $\mu_{\min } \leq \bar{\mu} \leq \mu_{\max }$ and $\bar{\mu} \leq 2 \mu_{\min }$.

3.1. Model problem: We consider the linear isotropic elasticity problem with discontinuous Lamé parameters across $\Gamma$, even though the following discussion and analysis can also be particularised to the Poisson equation, or any other elliptic problem with $H^{1}$-stability. We adopt a pure-displacement (irreducible) model [62]. For simplicity, we assume homogeneous Dirichlet boundary conditions on $\partial \Omega$, although non-homogeneous Dirichlet or Neumann boundary conditions can be considered too, using standard arguments. We also assume non-homogeneous (immersed) interface transmission conditions. According to this, the model problem [63, 64] seeks to find the displacement field $\boldsymbol{u}: \Omega^{+} \cup \Omega^{-} \rightarrow \mathbb{R}^{d}$ such that

$$
\begin{cases}-\boldsymbol{\nabla} \cdot \boldsymbol{\sigma}(\boldsymbol{u})=\boldsymbol{f} & \text { in } \Omega^{+} \cup \Omega^{-}, \\ \boldsymbol{u}=0 & \text { on } \partial \Omega, \\ \llbracket \boldsymbol{u} \rrbracket=\boldsymbol{j}_{\Gamma} & \text { on } \Gamma, \text { and } \\ \llbracket \boldsymbol{\sigma}(\boldsymbol{u}) \rrbracket \cdot \boldsymbol{n}^{+}=\boldsymbol{g}_{\Gamma} & \text { on } \Gamma,\end{cases}
$$

where $\varepsilon, \sigma: \Omega^{+} \cup \Omega^{-} \rightarrow \mathbb{R}^{d, d}$ are the strain tensor $\boldsymbol{\varepsilon}(\boldsymbol{u}) \doteq \frac{1}{2}\left(\boldsymbol{\nabla u}+\boldsymbol{\nabla} \boldsymbol{u}^{T}\right)$ and stress tensor $\boldsymbol{\sigma}(\boldsymbol{u})=2 \mu \boldsymbol{\varepsilon}(\boldsymbol{u})+$ $\lambda \operatorname{tr}(\boldsymbol{\varepsilon}(\boldsymbol{u})) \mathbf{I d}$; where Id denotes the identity matrix in $\mathbb{R}^{\mathrm{d}}$. Apart from that, we let $\boldsymbol{f} \in \boldsymbol{L}^{2}(\Omega)$ represent the body forces, whereas $\boldsymbol{j}_{\Gamma}$ and $\boldsymbol{g}_{\Gamma}$ denote the fixed jump and forcing terms on $\Gamma$. We assume that $\boldsymbol{j}_{\Gamma} \in \boldsymbol{H}_{00}^{1 / 2}(\Gamma)$ and $\boldsymbol{g}_{\Gamma} \in \boldsymbol{H}^{1 / 2}(\Gamma)$. We recall that $\boldsymbol{H}_{00}^{1 / 2}(\Gamma)$ is the subspace of functions in $\boldsymbol{H}^{1 / 2}(\Gamma)$, whose extension by zero on $\partial \boldsymbol{\Omega}$ is in $\boldsymbol{H}^{1 / 2}(\partial \boldsymbol{\Omega} \cup \Gamma)$ [61, Appendix A.2]. Since $\boldsymbol{j}_{\Gamma} \in \boldsymbol{H}_{00}^{1 / 2}(\Gamma)$, its extension by zero to $\partial \Omega^{\alpha}, \alpha \in\{+,-\}$, is bounded in $\boldsymbol{H}^{1 / 2}\left(\partial \Omega^{\alpha}\right)$, which we represent with $\boldsymbol{j}_{\partial \Omega^{\alpha}}$.

We assume the Lamé coefficients to be subdomain constant, i.e. $\lambda(\boldsymbol{x}) \doteq \lambda_{\alpha} \geq 0$ and $\mu(\boldsymbol{x}) \doteq \mu_{\alpha}>0$ for $x \in \Omega^{\alpha}, \alpha \in\{+,-\}$, but can have different values across $\Gamma$. Furthermore, we consider the Poisson ratio $v_{\alpha} \doteq$ $\lambda_{\alpha} /\left(2\left(\lambda_{\alpha}+\mu_{\alpha}\right)\right)$ is bounded away from $1 / 2$, i.e. the material is compressible. Since $\lambda_{\alpha}=2 v_{\alpha} \mu_{\alpha} /\left(1-2 v_{\alpha}\right)$, $\lambda_{\alpha}$ is bounded above by $\mu_{\alpha}$, i.e. $\lambda_{\alpha} \leq C \mu_{\alpha}, C>0$. Combined with the Cauchy-Schwarz inequality, it leads to the upper bound

$$
\int_{\Omega^{\alpha}} \boldsymbol{\sigma}(\boldsymbol{u}): \boldsymbol{\varepsilon}(\boldsymbol{v}) \mathrm{d} \Omega \lesssim \mu_{\alpha}\|\boldsymbol{\nabla} \boldsymbol{u}\|_{\mathbf{L}^{2}\left(\Omega^{\alpha}\right)}\|\boldsymbol{\nabla} \boldsymbol{v}\|_{\mathbf{L}^{2}\left(\Omega^{\alpha}\right)}, \quad \forall \boldsymbol{u}, \boldsymbol{v} \in \boldsymbol{H}^{1}\left(\Omega^{\alpha}\right) .
$$

On the other hand, letting $\mathcal{V} \doteq\left\{\boldsymbol{v} \in \boldsymbol{H}^{1}\left(\Omega^{ \pm}\right): \boldsymbol{v}=\mathbf{0}\right.$ on $\left.\partial \Omega\right\}$, we have the Korn inequality [65, (1.19)]

$$
\int_{\Omega} \boldsymbol{\sigma}(\boldsymbol{u}): \boldsymbol{\varepsilon}(\boldsymbol{u}) \mathrm{d} \Omega+\sum_{F \in \mathcal{F}_{h}} h_{T_{F}}^{-1}\|\llbracket \boldsymbol{u}\|\left\|_{\boldsymbol{L}^{2}(F)}^{2} \geq \sum_{\alpha \in\{+,-\}} C_{\sigma} C_{\Omega} \mu_{\alpha}\right\| \nabla \boldsymbol{u}^{\alpha} \|_{\boldsymbol{L}^{2}\left(\Omega^{\alpha}\right)}^{2}, \quad \forall \boldsymbol{u} \in \mathcal{V},
$$

where $C_{\Omega}>0$ is the related Korn constant. We can now use (3), (4) and the fact that $\boldsymbol{j}_{\Gamma} \in \boldsymbol{H}_{00}^{1 / 2}(\Gamma)$ to show that the weak form of (2) is well-posed. To this end, we let the decomposition $\boldsymbol{u} \doteq \boldsymbol{w}+\boldsymbol{h}_{\boldsymbol{j}} \in \boldsymbol{H}^{1}\left(\Omega^{ \pm}\right)$, such that the weak solution of (2) becomes: find $\boldsymbol{u}=\boldsymbol{w}+\boldsymbol{h}_{\boldsymbol{j}} \in \mathcal{V}$, where

$$
\begin{aligned}
& \boldsymbol{h}_{\boldsymbol{j}} \in \boldsymbol{H}^{1}\left(\Omega^{+}\right): \int_{\Omega^{+}} \boldsymbol{\sigma}\left(\boldsymbol{h}_{\boldsymbol{j}}\right): \boldsymbol{\varepsilon}(\boldsymbol{v}) \mathrm{d} \Omega=0, \quad \boldsymbol{h}_{\boldsymbol{j}}=\boldsymbol{j}_{\partial \Omega^{+}} \text {in } \partial \Omega^{+}, \quad \text { and } \\
& \boldsymbol{w} \in \boldsymbol{H}_{0}^{1}(\Omega): \int_{\Omega} \boldsymbol{\sigma}(\boldsymbol{w}): \boldsymbol{\varepsilon}(\boldsymbol{v}) \mathrm{d} \Omega=-\int_{\Omega^{+}} \boldsymbol{\sigma}\left(\boldsymbol{h}_{\boldsymbol{j}}\right): \boldsymbol{\varepsilon}(\boldsymbol{v}) \mathrm{d} \Omega+\int_{\Omega} \boldsymbol{f} \cdot \boldsymbol{v} \mathrm{d} \Omega+\int_{\Gamma} \boldsymbol{g}_{\Gamma} \cdot \boldsymbol{v} \mathrm{d} \Gamma,
\end{aligned}
$$

for all $\boldsymbol{v} \in \boldsymbol{H}_{0}^{1}(\Omega)$.

Continuity of the bilinear form in $\boldsymbol{H}^{1}\left(\Omega^{ \pm}\right)$is a direct consequence of (3). Since the jump term in (4) vanishes for $\boldsymbol{w} \in \boldsymbol{H}_{0}^{1}(\Omega)$, we can combine (4) with the first Poincaré-Friedrichs inequality to prove coercivity of (6). If we consider a continuous lifting of the Dirichlet data $\boldsymbol{j}_{\partial \Omega^{+}}$[61, Remark A.42], we can rewrite (5), as an 
homogeneous Dirichlet problem, and apply (4) in $\Omega^{+}$(again with null jump term). As a result, we can repeat the previous argument to show coercivity of (5) in $\Omega^{+}$. Thus, we can readily apply Lax-Milgram's lemma on (5), leading to $\left\|\boldsymbol{h}_{\boldsymbol{j}}\right\|_{\boldsymbol{H}^{1}\left(\Omega^{+}\right)} \lesssim\left\|\boldsymbol{j}_{\partial \Omega^{+}}\right\|_{\boldsymbol{H}^{1 / 2}\left(\partial \Omega^{+}\right)} \lesssim\left\|\boldsymbol{j}_{\Gamma}\right\|_{\boldsymbol{H}^{1 / 2}(\Gamma)}$. Finally, continuity of the right-hand side of (6) follows from the Cauchy-Schwarz inequality and a trace theorem:

$$
\begin{aligned}
-\int_{\Omega^{+}} \boldsymbol{\sigma}\left(\boldsymbol{h}_{\boldsymbol{j}}\right): \boldsymbol{\varepsilon}(\boldsymbol{v}) \mathrm{d} \Omega & \lesssim \mu_{+}^{1 / 2}\left\|\boldsymbol{j}_{\Gamma}\right\|_{\boldsymbol{H}^{1 / 2}(\Gamma)}\left\|\mu^{1 / 2} \boldsymbol{v}\right\|_{\boldsymbol{H}^{1}\left(\Omega^{+}\right)}, \\
\int_{\Omega} \boldsymbol{f} \cdot \boldsymbol{v} \mathrm{d} \Omega & \lesssim\left\|\mu^{-1 / 2} \boldsymbol{f}\right\|_{\boldsymbol{L}^{2}(\Omega)}\left\|\mu^{1 / 2} \boldsymbol{v}\right\|_{\boldsymbol{L}^{2}(\Omega)}, \\
\int_{\Gamma} \boldsymbol{g}_{\Gamma} \cdot \boldsymbol{v} \mathrm{d} \Gamma & \lesssim\left\|\bar{\mu}^{-1 / 2} \boldsymbol{g}_{\Gamma}\right\|_{\boldsymbol{L}^{2}(\Gamma)}\left\|\bar{\mu}^{1 / 2} \boldsymbol{v}\right\|_{\boldsymbol{L}^{2}(\Gamma)} \lesssim\left\|\bar{\mu}^{-1 / 2} \boldsymbol{g}_{\Gamma}\right\|_{\boldsymbol{H}^{1 / 2}(\Gamma)}\left\|\mu^{1 / 2} \boldsymbol{v}\right\|_{\boldsymbol{H}^{1}\left(\Omega^{ \pm}\right)}, \quad \forall \boldsymbol{v} \in \boldsymbol{H}_{0}^{1}(\Omega) .
\end{aligned}
$$

Combining all these results, existence and uniqueness of the weak solution to (2) is ensured by the Lax-Milgram theorem. Moreover, the problem is well-posed, since the unique solution is bounded by the data as follows:

$$
\left\|\mu^{1 / 2} \boldsymbol{u}\right\|_{\boldsymbol{H}^{1}\left(\Omega^{ \pm}\right)} \lesssim \mu_{+}^{1 / 2}\left\|\boldsymbol{j}_{\Gamma}\right\|_{\boldsymbol{H}^{1 / 2}(\Gamma)}+\left\|\mu^{-1 / 2} \boldsymbol{f}\right\|_{\boldsymbol{L}^{2}(\Omega)}+\left\|\bar{\mu}^{-1 / 2} \boldsymbol{g}_{\Gamma}\right\|_{\boldsymbol{H}^{1 / 2}(\Gamma)} .
$$

3.2. Discrete formulation. We consider as approximation space of $\mathcal{V}$ the aggregated FE space, see Section 2.3,

$$
\mathcal{V}_{h} \doteq\left\{\boldsymbol{v}_{h} \in \mathcal{V}_{\mathrm{ag}}^{+} \times \mathcal{V}_{\mathrm{ag}}^{-}: \boldsymbol{v}_{h}=\mathbf{0} \text { on } \partial \Omega\right\} .
$$

We consider an approximation of (6) with this discrete space, which reads:

$$
\boldsymbol{u}_{h} \in \mathcal{V}_{h}: a_{h}\left(\boldsymbol{u}_{h}, \boldsymbol{v}_{h}\right)=\ell_{h}\left(\boldsymbol{v}_{h}\right), \quad \forall \boldsymbol{v}_{h} \in \mathcal{V}_{h},
$$

where the global FE operators $a_{h}$ and $\ell_{h}$ are given by

$$
\begin{aligned}
a_{h}\left(\boldsymbol{u}_{h}, \boldsymbol{v}_{h}\right) \doteq & \int_{\Omega} \boldsymbol{\sigma}\left(\boldsymbol{u}_{h}\right): \boldsymbol{\varepsilon}\left(\boldsymbol{v}_{h}\right) \mathrm{d} \Omega \\
& +\sum_{F \in \mathcal{F}_{h}}\left[\frac{\beta \bar{\mu}}{h_{T_{F}}} \int_{F} \llbracket \boldsymbol{u}_{h} \rrbracket \cdot \llbracket \boldsymbol{v}_{h} \rrbracket \mathrm{d} \Gamma-\int_{F} \boldsymbol{n}^{+} \cdot\left\{\left\{\boldsymbol{\sigma}\left(\boldsymbol{v}_{h}\right)\right\}\right\} \cdot \llbracket \boldsymbol{u}_{h} \rrbracket \mathrm{d} \Gamma-\int_{F} \boldsymbol{n}^{+} \cdot\left\{\left\{\boldsymbol{\sigma}\left(\boldsymbol{u}_{h}\right)\right\}\right\} \cdot \llbracket \boldsymbol{v}_{h} \rrbracket \mathrm{d} \Gamma\right], \\
\ell_{h}\left(\boldsymbol{v}_{h}\right) \doteq & \int_{\Omega} \boldsymbol{f} \cdot \boldsymbol{v}_{h} \mathrm{~d} \Gamma \\
& +\sum_{F \in \mathcal{F}_{h}}\left[\frac{\beta \bar{\mu}}{h_{T_{F}}} \int_{F} \boldsymbol{j}_{\Gamma} \cdot \llbracket \boldsymbol{v}_{h} \rrbracket \mathrm{d} \Gamma-\int_{F} \boldsymbol{n}^{+} \cdot\left\{\left\{\boldsymbol{\sigma}\left(\boldsymbol{v}_{h}\right)\right\}\right\} \cdot \boldsymbol{j}_{\Gamma} \mathrm{d} \Gamma+\int_{F} \boldsymbol{g}_{\Gamma} \cdot\left(w_{-} \boldsymbol{v}_{h}^{+}+w_{+} \boldsymbol{v}_{h}^{-}\right) \mathrm{d} \Gamma\right] .
\end{aligned}
$$

We observe that $a_{h}$ and $\ell_{h}$ contain the usual terms in Nitsche's formulations, i.e. terms that weakly impose the interface conditions, symmetrizing terms and stabilization terms. The latter terms are those premultiplied by $\beta$, which has to be large-enough to ensure coercivity of the bilinear form $a_{h}$. Furthermore, the above formulation is consistent, by the following result:

Lemma 3.1 (Consistency). Let $\boldsymbol{u} \in \boldsymbol{H}^{2}\left(\Omega^{ \pm}\right) \cap \mathcal{V}$ solve (2). Then, it holds $a_{h}\left(\boldsymbol{u}, \boldsymbol{v}_{h}\right)=\ell_{h}\left(\boldsymbol{v}_{h}\right), \forall \boldsymbol{v}_{h} \in \mathcal{V}_{h}$.

Proof. Since $\boldsymbol{u}$ solves problem (2) (in a weak sense), integration by parts leads to:

$$
\begin{aligned}
\int_{\Omega} \boldsymbol{\sigma}(\boldsymbol{u}): \boldsymbol{\varepsilon}\left(\boldsymbol{v}_{h}\right) \mathrm{d} \Omega & =-\int_{\Omega^{+} \cup \Omega^{-}} \boldsymbol{v}_{h} \cdot \boldsymbol{\nabla} \cdot \boldsymbol{\sigma}(\boldsymbol{u}) \mathrm{d} \Omega \\
& +\int_{\Gamma} \boldsymbol{n}^{+} \cdot\{\{\boldsymbol{\sigma}(\boldsymbol{u})\}\} \cdot \llbracket \boldsymbol{v}_{h} \rrbracket \mathrm{d} \Gamma+\int_{\Gamma} \boldsymbol{n}^{+} \cdot \llbracket \boldsymbol{\sigma}(\boldsymbol{u}) \rrbracket \cdot\left(w^{-} \boldsymbol{v}_{h}^{+}+w^{+} \boldsymbol{v}_{h}^{-}\right) \mathrm{d} \Gamma,
\end{aligned}
$$

for any $\boldsymbol{v}_{h} \in \mathcal{V}_{h}$. Combining this result with $-\boldsymbol{\nabla} \cdot \boldsymbol{\sigma}(\boldsymbol{u})=\boldsymbol{f}, \llbracket \boldsymbol{u} \rrbracket=\boldsymbol{j}_{\Gamma}$ and $\boldsymbol{n}^{+} \cdot \llbracket \boldsymbol{\sigma}(\boldsymbol{u}) \rrbracket=\boldsymbol{g}_{\Gamma}$, we can check that all terms in the discrete formulation (7) cancel out.

For the sake of proving coercivity, we need the following auxiliary result.

Lemma 3.2. Let $T \in \mathcal{T}_{h, \mathrm{~W}}^{\alpha}$ and $\boldsymbol{u}_{T} \in Q_{q}(T)$. There exists $C_{\eta_{0}}>0$, dependent on the well-posedness threshold $\eta_{0}$, such that $\left\|\boldsymbol{u}_{T}\right\|_{\boldsymbol{L}^{2}(T)}^{2} \leq C_{\eta_{0}}\left\|\boldsymbol{u}_{T}\right\|_{\boldsymbol{L}^{2}\left(T \cap \Omega^{\alpha}\right)}^{2}, \alpha \in\{+,-\}$.

Proof. The proof is direct for interior well-posed cells; we restrict ourselves to well-posed cut cells. Let us consider a cell $T$ and its interior portion $T \cap \Omega$. Using the inverse of the geometrical map, which maps $T$ into the reference cell $\hat{T}$, one can map the interior portion to the reference cell, which is represented with $\hat{T}_{\text {in }}$. It is easy to check that meas $d\left(\hat{T}_{\text {in }}\right) \geq C \eta_{0}$ meas $_{d}(\hat{T})$. In fact, the constant is 1 for affine maps. $\|\cdot\|_{\mathbf{L}^{2}\left(\hat{T}_{\text {in }}\right)}^{2}$ is a norm for $Q_{q}(\hat{T})$, since a polynomial that vanishes in a domain of non-zero measure is equal to zero. We prove the result by using the equivalence of norms in finite-dimensional vector spaces and a scaling argument. 
Given $T \in \mathcal{T}_{h, \mathrm{~A}}^{\alpha}, \alpha \in\{+,-\}$, let us denote by $T_{1}, \ldots, T_{n_{T}^{\alpha}}, n_{T}^{\alpha} \geq 1$, the set of constraining well-posed cells of $T$ in $\mathcal{T}_{h, \mathrm{~W}}^{\alpha}$, i.e. the set of well-posed cells that constrain at least one DOF of $T$ in $\mathcal{T}_{h \text {, }}^{\alpha}$. Given $F \in \mathcal{F}_{h}$, we recall that $T_{F}^{\alpha}$ is the cell in $\mathcal{T}_{h, \mathrm{~A}}^{\alpha}$ satisfying $F \cap \overline{\Omega^{\alpha}} \subset \overline{T_{F}^{\alpha}}$. Hence, we define $\Omega_{T_{F}^{\alpha}} \doteq \Omega^{\alpha} \cap\left(T_{F}^{\alpha} \cup \bigcup_{i=1}^{n_{T}^{\alpha}} T_{i}\right)$. With this notation, we can state an inequality for discrete functions in cut cells (see [50, Lemma A.7]):

$$
\left\|\boldsymbol{\nabla} \boldsymbol{v}_{h}^{\alpha}\right\|_{\boldsymbol{L}^{2}(F)}^{2} \lesssim C_{\eta_{0}} h_{T_{F}^{\alpha}}^{-1}\left\|\boldsymbol{\nabla} \boldsymbol{v}_{h}^{\alpha}\right\|_{\boldsymbol{L}^{2}\left(\Omega_{\left.T_{F}^{\alpha}\right)}^{\alpha}\right.}^{2}, \quad \alpha \in\{+,-\}, \quad \forall \boldsymbol{v}_{h} \in \mathcal{V}_{h}, \quad \forall F \in \mathcal{F}_{h}
$$

We also make use of the following inequality for continuous functions on cut cells (see [28]):

$$
\|\psi\|_{L^{2}(\partial(\Omega \cap T))}^{2} \lesssim h_{T}^{-1}\|\psi\|_{L^{2}(\Omega \cap T)}^{2}+h_{T}|\psi|_{H^{1}(\Omega \cap T)}^{2}, \quad \forall \psi \in H^{1}(\Omega \cap T),
$$

where $\partial(\Omega \cap T)$ is the boundary of $\Omega \cap T$. $^{2}$

Let us define the space $\mathcal{V}(h) \doteq \mathcal{V}_{h}+\boldsymbol{H}^{2}\left(\Omega^{ \pm}\right) \cap \mathcal{V}$. We endow $\mathcal{V}(h)$ with the broken norm:

$$
\|v\|_{\mathcal{V}(h)}^{2} \doteq \sum_{\alpha \in\{+,-\}} \mu_{\alpha}\left\|\nabla^{\alpha}\right\|_{L^{2}\left(\Omega^{\alpha}\right)}^{2}+\sum_{F \in \mathcal{F}_{h}} \frac{\bar{\mu}}{h_{T_{F}}}\|\llbracket v\|\left\|_{\boldsymbol{L}^{2}(F)}^{2}+\sum_{\alpha \in\{+,-\}} \sum_{T \in \mathcal{T}_{h, \mathrm{~A}}^{\alpha}} \mu_{\alpha} h_{T}^{2}\right\| \boldsymbol{v} \|_{\boldsymbol{H}^{2}\left(T \cap \Omega^{\alpha}\right)}^{2} .
$$

It can be checked that $\|\boldsymbol{v}\|_{\boldsymbol{L}^{2}(\Omega)} \lesssim\|v\|_{\mathcal{V}(h)}$, for $\boldsymbol{v} \in \mathcal{V}(h)$, see, e.g. [38, Lemma 5.8]. The following lemma restricted to the discrete space $\mathcal{V}_{h}$ provides the well-posedness of the discrete problem. Its extension to $\mathcal{V}(h)$ will be required in the convergence analysis.

Lemma 3.3 (Well-posedness). The bilinear form in the discrete formulation (7) satisfies the following properties uniformly w.r.t. the mesh size $h$ of the background mesh and interface intersection:

(i) Coercivity:

$$
a_{h}\left(\boldsymbol{u}_{h}, \boldsymbol{u}_{h}\right) \gtrsim\left\|\boldsymbol{u}_{h}\right\|_{\mathcal{V}(h)}^{2}, \quad \forall \boldsymbol{u}_{h} \in \mathcal{V}_{h},
$$

if $\beta>C$, for some (large-enough) positive constant $C$.

(ii) Continuity:

$$
a_{h}(\boldsymbol{u}, \boldsymbol{v}) \lesssim\|\boldsymbol{u}\|_{\mathcal{V}(h)}\|\boldsymbol{v}\|_{\mathcal{V}(h)}, \quad \forall \boldsymbol{u}, \boldsymbol{v} \in \mathcal{V}(h) .
$$

Therefore, there exists a unique solution to problem (7).

Proof. By definition of the bilinear form $a_{h}$ and (4), we have that

$$
a_{h}\left(\boldsymbol{u}_{h}, \boldsymbol{u}_{h}\right) \gtrsim \sum_{\alpha \in\{+,-\}} C_{\sigma} C_{\Omega} \mu_{\alpha}\left\|\nabla \boldsymbol{u}_{h}^{\alpha}\right\|_{\boldsymbol{L}^{2}\left(\Omega^{\alpha}\right)}^{2}+\sum_{F \in \mathcal{F}_{h}} \frac{\beta \bar{\mu}}{h_{T_{F}}}\left\|\llbracket \boldsymbol{u}_{h} \rrbracket\right\|_{\boldsymbol{L}^{2}(F)}^{2}-2 \sum_{F \in \mathcal{F}_{h}} \int_{F} \boldsymbol{n}^{+} \cdot\left\{\left\{\boldsymbol{\sigma}\left(\boldsymbol{u}_{h}\right)\right\}\right\} \cdot \llbracket \boldsymbol{u}_{h} \rrbracket \mathrm{d} \Gamma,
$$

for any $\boldsymbol{u}_{h} \in \mathcal{V}_{h}$. In order to prove coercivity, we have to bound the indefinite term. Let us pick an arbitrary $\boldsymbol{u}_{h} \in \mathcal{V}_{h}$. Using the fact that $w_{\alpha} \mu_{\alpha}=\bar{\mu}$, Cauchy-Schwarz and triangle inequalities and (8), we get

$$
\left\|\boldsymbol{n}^{+} \cdot\left\{\left\{\boldsymbol{\sigma}\left(\boldsymbol{u}_{h}\right)\right\}\right\}\right\|_{\boldsymbol{L}^{2}(F)}^{2} \lesssim \sum_{\alpha \in\{+,-\}} w_{\alpha}^{2} \mu_{\alpha}^{2}\left\|\nabla \boldsymbol{u}_{h}^{\alpha}\right\|_{\boldsymbol{L}^{2}(F)}^{2}=\bar{\mu}^{2} \sum_{\alpha \in\{+,-\}}\left\|\nabla \boldsymbol{u}_{h}^{\alpha}\right\|_{\boldsymbol{L}^{2}(F)}^{2} \leq C_{\eta_{0}} \bar{\mu} \sum_{\alpha \in\{+,-\}} \frac{\mu_{\alpha}}{h_{T_{F}^{\alpha}}}\left\|\boldsymbol{\nabla} \boldsymbol{u}_{h}^{\alpha}\right\|_{\boldsymbol{L}^{2}\left(\Omega_{T_{F}^{\alpha}}\right)}^{2} .
$$

Usage of the Cauchy-Schwarz and Young inequalities and the previous result leads to

$$
\begin{aligned}
\left|2 \int_{F} \boldsymbol{n}^{+} \cdot\left\{\left\{\boldsymbol{\sigma}\left(\boldsymbol{u}_{h}\right)\right\}\right\} \cdot \llbracket \boldsymbol{u}_{h} \rrbracket \mathrm{d} \Gamma\right| & \lesssim \frac{h_{T_{F}}}{\gamma \bar{\mu}}\left\|\boldsymbol{n}^{+} \cdot\left\{\left\{\boldsymbol{\sigma}\left(\boldsymbol{u}_{h}\right)\right\}\right\}\right\|_{\boldsymbol{L}^{2}(F)}^{2}+\frac{\gamma \bar{\mu}}{h_{T_{F}}}\left\|\llbracket \boldsymbol{u}_{h} \rrbracket\right\|_{\boldsymbol{L}^{2}(F)}^{2} \\
& \lesssim C_{\eta_{0}} \sum_{\alpha \in\{+,-\}} \frac{\mu_{\alpha}}{\gamma}\left\|\boldsymbol{\nabla} \boldsymbol{u}_{h}^{\alpha}\right\|_{\boldsymbol{L}^{2}\left(\Omega_{\left.T_{F}^{\alpha}\right)}^{2}\right.}^{2}+\frac{\gamma \bar{\mu}}{h_{T_{F}}}\left\|\llbracket \boldsymbol{u}_{h} \rrbracket\right\|_{\boldsymbol{L}^{2}(F)}^{2}, \quad \forall \boldsymbol{u}_{h} \in \mathcal{V}_{h},
\end{aligned}
$$

with $\gamma>0$ an arbitrary positive constant. Combining (10) and (12), and using the fact that the number of neighbouring cells is bounded, we obtain:

$$
a_{h}\left(\boldsymbol{u}_{h}, \boldsymbol{u}_{h}\right) \gtrsim\left(C_{\sigma} C_{\Omega}-\frac{C_{\eta_{0}}}{\gamma}\right) \sum_{\alpha \in\{+,-\}} \mu_{\alpha}\left\|\nabla \boldsymbol{u}_{h}^{\alpha}\right\|_{\boldsymbol{L}^{2}\left(\Omega^{\alpha}\right)}^{2}+\left(1-\frac{\gamma}{\beta}\right) \sum_{F \in \mathcal{F}_{h}} \frac{\beta \bar{\mu}}{h_{T_{F}}}\left\|\llbracket \boldsymbol{u}_{h} \rrbracket\right\|_{\boldsymbol{L}^{2}(F)}^{2} .
$$

Let us pick $\gamma=\frac{2 C_{\eta_{0}}}{C_{\sigma} C_{\Omega}}$. Assuming $\beta \geq 2 \gamma$, the terms in the right-hand side are positive. In order to check that $a_{h}\left(\boldsymbol{u}_{h}, \boldsymbol{u}_{h}\right)$ is also a bound for the $\boldsymbol{H}^{2}$ broken semi-norm in $\|\cdot\|_{\mathcal{V}(h)}$, we proceed as follows. The local discrete inverse inequality $\left\|\nabla \xi_{h}\right\|_{L^{2}\left(T \cap \Omega^{\alpha}\right)} \leq C h^{-1}\left\|\xi_{h}\right\|_{L^{2}(T)}$ can readily be applied to finite element functions (and its gradients) in AgFE spaces (see, e.g. [38, (12)]). On the other hand, by Lemma 3.2 we have

\footnotetext{
${ }^{2}$ We note that the proof in [28] assumes that $\Omega \cap T$ is connected, together with the assumption that $\Gamma$ has a bounded curvature. The connected intersection can be handled either replicating cells (as commented above) or assuming a fine enough mesh.
} 
that $\left\|\xi_{h}\right\|_{\boldsymbol{L}^{2}(T)} \leq C\left\|\boldsymbol{\xi}_{h}\right\|_{\boldsymbol{L}^{2}\left(T \cap \Omega^{\alpha}\right)}$. As a result, we have that $h_{T}\left|\boldsymbol{v}_{h}\right|_{\boldsymbol{H}^{2}\left(T \cap \Omega^{\alpha}\right)} \leq C\left\|\nabla \boldsymbol{v}_{h}\right\|_{\boldsymbol{L}^{2}\left(T \cap \Omega^{\alpha}\right)}$, for any $v_{h} \in \mathcal{V}_{h}$. Hence, bilinear form $a_{h}$ satisfies coercivity; it is non-singular.

In order to prove continuity, we need a continuous version of (11) for functions in $\boldsymbol{H}^{2}\left(\Omega^{ \pm}\right) \cap \mathcal{V}$. Using (9), we get the sought-after bound:

$$
\left\|\boldsymbol{n}^{+} \cdot\{\{\boldsymbol{\sigma}(\boldsymbol{u})\}\}\right\|_{\boldsymbol{L}^{2}(F)}^{2} \lesssim \bar{\mu}^{2} \sum_{\alpha \in\{+,-\}}\left\|\nabla \boldsymbol{u}^{\alpha}\right\|_{\boldsymbol{L}^{2}(F)}^{2} \lesssim \bar{\mu} \sum_{\alpha \in\{+,-\}}\left(\frac{\mu_{\alpha}}{h_{T_{F}^{\alpha}}}\left\|\nabla \boldsymbol{u}^{\alpha}\right\|_{\boldsymbol{L}^{2}\left(T_{F}^{\alpha} \cap \Omega^{\alpha}\right)}^{2}+\mu_{\alpha} h_{T_{F}^{\alpha}}\left|\boldsymbol{u}^{\alpha}\right|_{\boldsymbol{H}^{2}\left(T_{F}^{\alpha} \cap \Omega^{\alpha}\right)}^{2}\right) .
$$

It follows that continuity is a consequence of (3), (11) for discrete functions in $\mathcal{V}_{h}$, (13) for functions in $\boldsymbol{H}^{2}\left(\Omega^{ \pm}\right) \cap \mathcal{V}$, and the Cauchy-Schwarz inequality. Since the problem is finite-dimensional and the corresponding linear system matrix is non-singular, there exists a unique solution to problem (7).

Let us assume that the background mesh $\mathcal{T}_{h}$ is quasi-uniform, with characteristic size $h \doteq \max _{T \in \mathcal{T}_{h}} h_{T}$. We adopt now an extended Scott-Zhang interpolant $\Pi_{h}^{\mathrm{SZ}}: \mathcal{V} \rightarrow \mathcal{V}_{h}$ given by $\Pi_{h}^{\mathrm{SZ}}(\boldsymbol{u})=\left\{\Pi_{h,+}^{\mathrm{SZ}}(\boldsymbol{u}), \Pi_{h,-}^{\mathrm{SZ}}(\boldsymbol{u})\right\}$ with $\Pi_{h, \alpha}^{\mathrm{SZ}}(\boldsymbol{u}) \in \mathcal{V}_{\mathrm{ag}}^{\alpha}, \alpha \in\{+,-\}$, defined in [51]. The local approximability property in [51, Theorem 4.4] and the trace inequality (9) applied to $\psi=\boldsymbol{u}^{\alpha}-\Pi_{h, \alpha}^{\mathrm{SZ}}(\boldsymbol{u})$ yield the following result.

Proposition 3.4. If $\boldsymbol{u} \in \boldsymbol{H}^{m}\left(\Omega^{ \pm}\right), m \geq 2$, and the order of $\mathcal{V}_{h}$ is greater or equal than $m-1$, then

$$
\left\|\boldsymbol{u}-\Pi_{h}^{\mathrm{SZ}}(\boldsymbol{u})\right\|_{\mathcal{V}(h)} \lesssim h^{m-1}|\boldsymbol{u}|_{\boldsymbol{H}^{m}\left(\Omega^{ \pm}\right)} .
$$

In order to prove a priori error estimates, we must assume additional regularity on the solution. For $\Omega$ being a convex polygon, $\Gamma$ of class $C^{2}$ and $\boldsymbol{g}_{\Gamma} \in \boldsymbol{H}_{00}^{1 / 2}(\Gamma)$, the interface problem enjoys smoothing properties and its solution $\boldsymbol{u} \in \boldsymbol{H}^{2}\left(\Omega^{ \pm}\right) \cap \mathcal{V}$ (see [66]). Neglecting the geometrical error, the consistency in Lemma 3.1, well-posedness in Lemma 3.3 and the approximability property in Proposition 3.4 can be combined to prove an estimate in the $\mathcal{V}(h)$ norm. Furthermore, under the previous assumptions, a duality argument analogous to [28, Theorem 6] can be used to obtain the $L^{2}$ estimate. The geometrical error in the approximation could be incorporated into the discussion with the same ideas as, e.g. in [66].

Proposition 3.5. If $\boldsymbol{u} \in \boldsymbol{H}^{m}\left(\Omega^{ \pm}\right), m \geq 2$, is the solution of (5)-(6) and $\boldsymbol{u}_{h} \in \mathcal{V}_{h}$ is the solution of (7), with the order of $\mathcal{V}_{h}$ greater or equal than $m-1$, then

$$
\left\|\boldsymbol{u}-\boldsymbol{u}_{h}\right\|_{\mathcal{V}(h)} \lesssim h^{m-1}|\boldsymbol{u}|_{\boldsymbol{H}^{m}\left(\Omega^{ \pm}\right)}, \quad\left\|\boldsymbol{u}-\boldsymbol{u}_{h}\right\|_{\boldsymbol{L}^{2}(\Omega)} \lesssim h^{m}|\boldsymbol{u}|_{\boldsymbol{H}^{m}\left(\Omega^{ \pm}\right)} .
$$

\section{NUMERICAL EXPERIMENTS}

Our goal, in this section, is to analyse numerically the accuracy, optimality, robustness and performance of $h$ AgFEM for interface elliptic BVPs. We consider as model problems the Poisson and linear elasticity equations, with non-homogeneous Dirichlet conditions on the external boundary and discretised with the variational formulation detailed in Section 3.2. We describe first the experimental setup in Section 4.1, consisting of several manufactured problems defined in a set of complex geometries. We lay out next the experimental environment of the $h$-AgFEM parallel implementation in FEMPAR [56]. After these preliminaries, we move to report and discuss the numerical results of three different sets of experiments: convergence tests in Section 4.3, material contrast and cut location robustness tests in Section 4.4 and, finally, weak scaling tests in Section 4.5.

4.1. Experimental benchmarks. Numerical tests consider the variational formulation of Section 3.2, with nonhomogeneous Dirichlet boundary conditions, applied to the Poisson and linear elasticity problems. Although exposition was restricted to linear elasticity, the formulation for the Poisson equation can be easily derived, as a particular case. This leads to an analogous formulation to the ones in [17, 28, 52]. We observe that, with little effort, the Poisson equation inherits well-posedness and approximability results proven in Section 3. Moreover, harmonic weights become $w^{+}=\frac{k^{-}}{k^{+}+k^{-}}$and $w^{-}=\frac{k^{+}}{k^{+}+k^{-}}$, where $k^{\alpha}>0, \alpha \in\{+,-\}$, represents the subdomain-wise constant diffusion coefficient.

Numerical experiments are carried out on both serial and parallel, distributed-memory, environments. We generally report parallel results; serial ones are only shown when informing about condition numbers. We also observe that parallelisation of interface AgFEM basically reuses ideas that have already been covered in [54]. In addition, all examples run on background Cartesian grids, endowed with standard isotropic 1:4 (2D) or 1:8 (3D) refinement rules; also known as quadtrees (2D) or octrees (3D) [60]. We have also addressed in [50] how to build $\mathrm{AgFE}$ spaces on top of these (generally) nonconforming meshes. In the experiments, we consider both uniform and $h$-adaptive refinements. The latter follow an iterative Adaptive mesh refinement and coarsening (AMR) process [50] that exploits the Li and Bettess convergence (or acceptability) criterion [67, 68]. As usual, the goal of the procedure is to find an optimal mesh, that minimises the number of cells required to achieve a given 
discretisation error. Nonetheless, we remark that remeshing is not driven by a posterior error estimation, since we can compute the exact error in all cases studied, and we do not consider the geometrical error in approximating the interface. In contrast to [50], we use the relative energy norm error in the acceptability criterion to eliminate the influence of material contrast. Seeking to ensure stability, without superfluous aggregation, that degrades accuracy and conditioning [50], the well-posedness threshold $\eta_{0}$ to isolate badly-cut cells is set to 0.25 .

The FE approximation space for all experiments is $\mathcal{V}_{h}$, described in Section 3.2, as the single-interface version of the general $n$-interface $\mathcal{V}_{h \text {,ag }}$ in Section 2.3. Henceforth, we refer to $\mathcal{V}_{h}$ simply as the AgFE space. We employ both first and second order Lagrangian finite elements. Following discussion in Section 3.2, the coercivity coefficient is given by $\beta=10.0 q^{2}$, where $q$ is the FE order; this value is enough to ensure wellposedness for all the tests below. Apart from that, robustness tests, in Section 4.4, additionally consider a standard FE (or StdFE) space defined by $\mathcal{V}_{h}^{\text {std }} \doteq \mathcal{V}_{h, \mathrm{~A}}^{+} \times \mathcal{V}_{h, \mathrm{~A}}^{-}$. Although $\mathcal{V}_{h}^{\text {std }}$ is stable to cut location, under suitable mesh and interface regularity conditions [28], it leads to much more ill-conditioned systems than the $\mathrm{AgFE}$ space [37]. For this reason, usage of StdFE space is merely intended to provide a numerical reference to assess the condition number of AgFE space. When using the StdFE space, the $\beta$ coefficient at each (well- or ill-posed) cut cell is computed by solving a generalised eigenvalue problem, detailed in [38, Section 4.2].

The physical domain in all cases is a cuboid (of varying sizes), but the physical interface dividing the two phases is a non-trivial surface, described as the 0-level set of a (piecewise-)smooth function. We consider eight different level-set interfaces: (a) a circle, (b) a flower and (c) a "pacman" shape, in 2D; (d) a cylinder, (e) a popcorn flake, (f) a spiral, (g) a popcorn flake without a wedge (popcorn pacman) and (h) a gyroid, in 3D. All these geometries are covered in the literature [17, 41]; they are typically chosen to examine the behaviour of unfitted FE methods. We consider linear approximations of the embedded interfaces; in the numerical results, the geometrical error does not affect global optimality (of quadratic FEs). However, in general, high-order geometrical approximations of the interface are required in order to retain optimality of AgFEM with high-order FEs. For illustration purposes, descriptive figures of the considered interfaces (or the interior region that they enclose) are drawn along the convergence plots of Section 4.3. Besides, the geometry for the gyroid problem is represented in Figure 4.

We study four different analytical benchmarks; all of them are derived with the so-called method of manufactured solutions [69], i.e. we propose a solution of the problem with known analytical solution and then we compute source term and interface conditions from the governing equations (2). For the Poisson problem we consider a benchmark for verification (convergence tests), namely the (1) out-FE-space benchmark. We add two more Poisson benchmarks, that correspond to adapted versions of two classical $h p$-FEM problems, the (2) Fichera-corner and (3) single-shock problems. For linear elasticity, we address the (4) cylindrical inclusion problem in [70]. Let us next provide the analytical expressions of the solution function for each case.

- The out-FE-space benchmark is adapted from [36] and applied to several interface geometries. The solution is given by $u(q, \mathbf{x}): \Omega \subset \mathbb{R}^{d} \rightarrow \mathbb{R}$ and $q \in \mathbb{N}$ such that

$$
u(q ; \mathbf{x}) \doteq \begin{cases}\frac{k^{+}-k^{-}+\left(3 k^{-}+k^{+}\right) x}{4 k^{+}\left(k^{-}+k^{+}\right)}-\frac{x^{q+1}}{(q+1) k^{+}}, & \text {if } \mathbf{x} \in \Omega^{+}, \\ \frac{\left(3 k^{-}+k^{+}\right) x}{4 k^{-}\left(k^{-}+k^{+}\right)}-\frac{x^{q+1}}{(q+1) k^{-}}, & \text {if } \mathbf{x} \in \Omega^{-} .\end{cases}
$$

In our case, we take $q$ as the FE interpolation order, then $u \notin \mathcal{V}_{h}$. Moreover, $u$ is discontinuous across $\Gamma$, but the jump of normal fluxes is null, i.e. $\llbracket k \nabla u \rrbracket \cdot \boldsymbol{n}^{+}=0$.

- The Fichera-corner benchmark is adapted from [71] and applied to the pacman and popcorn-pacman interface shapes. The solution $u(r, \theta, z): \Omega \subset \mathbb{R}^{d} \rightarrow \mathbb{R}$ in cylindrical coordinates is

$$
u^{\alpha}(r, \theta, z) \doteq r^{\omega^{\alpha}} \sin \omega^{\alpha} \theta, \alpha \in\{+,-\}, \omega^{-}=2 / 3, \omega^{+}=4 .
$$

Numerical solution of (15) in the popcorn flake without a wedge is represented in Figure 5. We observe that the problem has fully non-homogeneous interface conditions. Furthermore, $u^{+}$is smooth, whereas derivatives of $u^{-}$are singular at the $r=0$ axis; specifically, $u^{-} \in H^{1+\frac{2}{3}}\left(\Omega^{-}\right)$. When only approximating $u^{-}$, convergence rates of the energy norm with uniform refinements are limited by regularity; they decrease at a rate $O\left(h^{2 / 3}\right)$. Optimal convergence rates can be restored with $h$-adaptivity [71]. In Section 4.3, we argue that, even though $u$ does not explicitly depend on the diffusion coefficients, material contrast determines whether convergence behaviour of $u$ (in the energy norm) is dictated by regularity of $u^{+}$or $u^{-}$.

- The single-shock benchmark is also adapted from [71] and applied to the gyroid interface. The solution $u(r): \Omega \subset \mathbb{R}^{d} \rightarrow \mathbb{R}$ is

$$
u(r) \doteq \arctan \left(\tau\left(r-r_{0}\right)\right), \tau=60, r=\left\|\mathbf{x}-\mathbf{x}_{0}\right\|_{2}, r_{0}=2.5, \mathbf{x}_{0}=\left(x_{0}, y_{0}, z_{0}\right)=(-1,-1,1),
$$




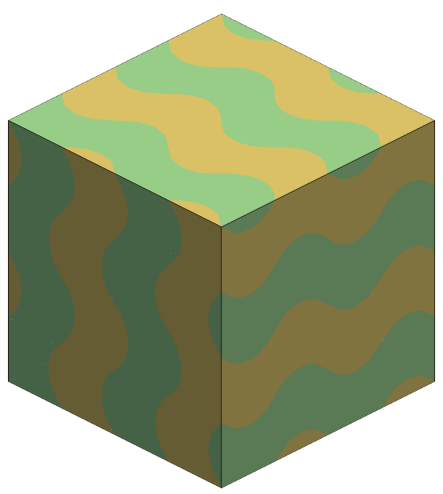

(A)

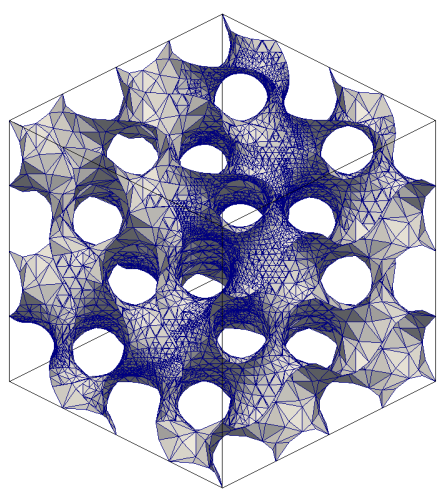

(D)

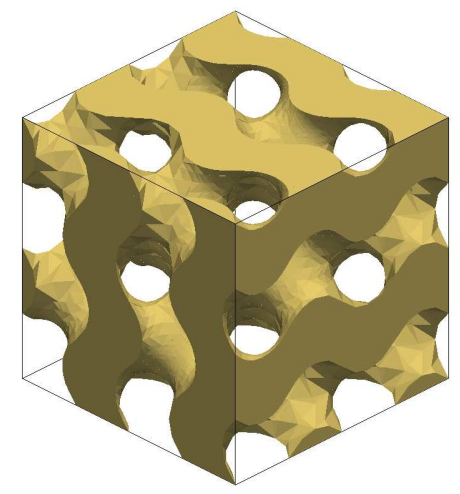

(B)

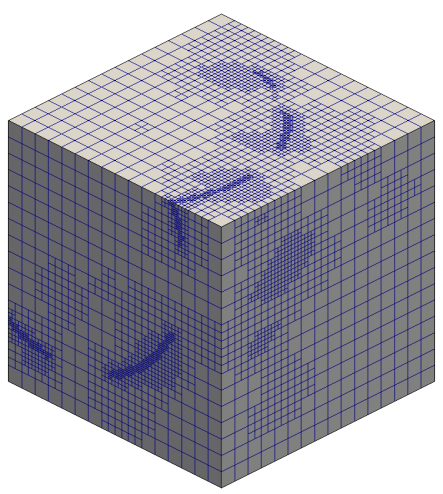

(E)

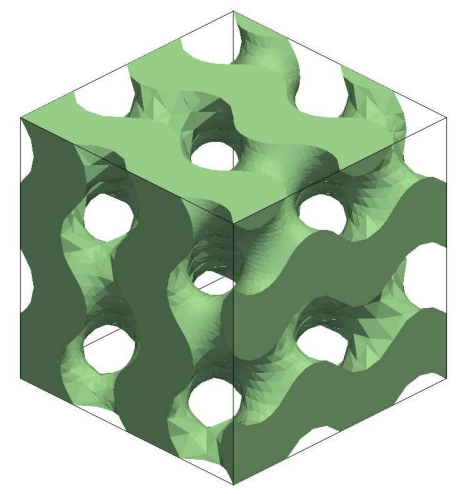

(c)

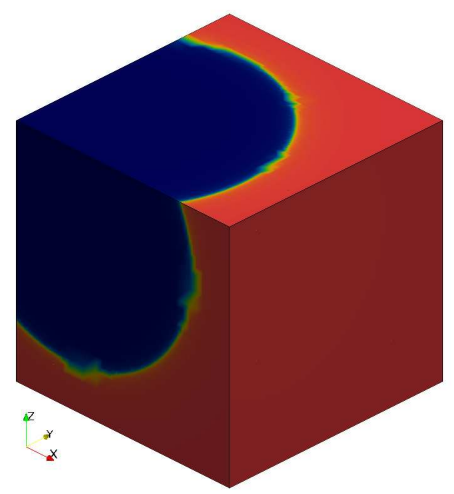

(F)

FIGURE 4. The gyroid interface and single-shock benchmark. The top three figures represent the two regions (together and one-by-one) divided by the gyroid level-set function on the region $[-2,2]^{3}$. The bottom three figures represent the mesh and solution of the single-shock equation (16) with $k^{+} / k^{-} \neq 1$ on a given $h$-adaptive mesh: the discrete approximated interface in Figure 4D, the mesh in Figure 4E and the solution in Figure 4F. Different mesh resolution is due to dependency of the energy norm error on material contrast.

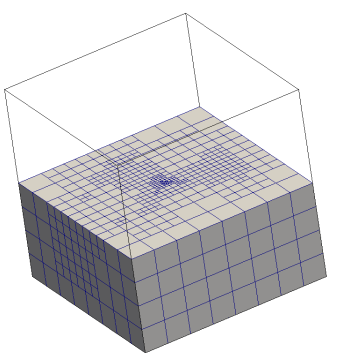

(A) $k^{+} / k^{-}=1$

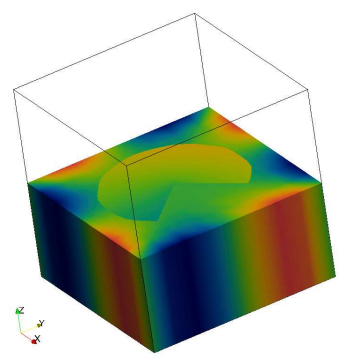

(в) $k^{+} / k^{-}=1$

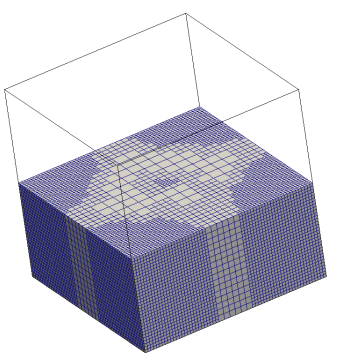

(c) $k^{+} / k^{-}=10^{6}$

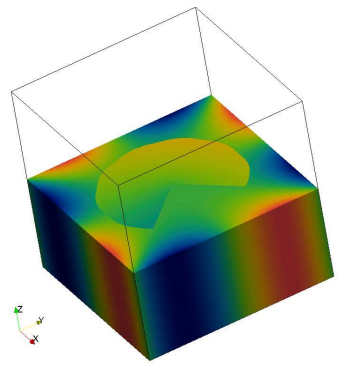

(D) $k^{+} / k^{-}=10^{6}$

FIGURE 5. The Fichera-corner benchmark (15) on the popcorn-pacman interface in two different situations. We only show mesh and solution at the bottom half of the simulated cube, to show the results on the $z=0$ plane. Material contrast determines which of the solution sides dominate the numerical error. In the two left plots, $k^{+} / k^{-}=1$ leads to a situation where error and, thus, refinements concentrate in $\Omega^{-}$. Conversely, in the two right plots, $k^{+} / k^{-}=10^{6}$ yields higher errors and mesh refinements in $\Omega^{+}$.

where $\|\cdot\|_{2}$ denotes the Euclidean norm. Numerical solution of (16) in the gyroid is represented in Figure 4F. As in the previous benchmark (15), the analytical solution does not depend on the material parameters, but numerical error (in the energy norm) does. Apart from that, $u$ is smooth in $\Omega$, although it sharply varies in the neighbourhood of the shock, and continuous across $\Gamma$, although with a kink if $k^{+} \neq k^{-}$. We notice that the shock may intersect $\Gamma$, e.g. it crosses several times the gyroid 0-level set. 
- The cylindrical inclusion benchmark is applied to a cylindrical interface. It adapts the linear elasticity problem in [70, Section 7.3]. The displacement in cylindrical coordinates is given by:

$$
u_{r}(r) \doteq\left\{\begin{array}{ll}
{\left[\left(1-\frac{b^{2}}{a^{2}}\right) c+\frac{b^{2}}{a^{2}}\right] r,} & 0 \leq r<a, \\
\left(r-\frac{b^{2}}{r}\right) c+\frac{b^{2}}{r}, & a \leq r \leq b .
\end{array}, \quad u_{\theta} \equiv 0, \quad u_{z} \equiv 0,\right.
$$

where $a=0.4, b=2.0$ and

$$
c=\frac{\left(\lambda_{-}+\mu_{-}+\mu_{+}\right) b^{2}}{\left(\lambda_{+}+\mu_{+}\right) a^{2}+\left(\lambda_{-}+\mu_{-}\right)\left(b^{2}-a^{2}\right)+\mu_{+} b^{2}} .
$$

In the experiments, $\Omega \subset\{0 \leq r<b\}$ and $\Omega^{-}=\{0 \leq r<a\}$. The numerical solution is represented in Figure 6 . As in (16), $u$ is continuous across $\Gamma$, but it has a kink if material properties are discontinuous.

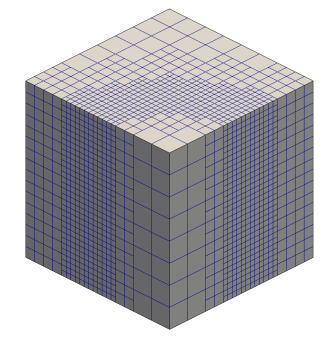

(A)

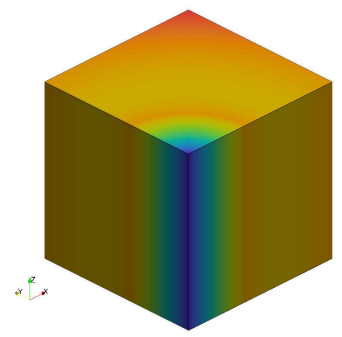

(B)

FigURE 6. Error-driven adaptive mesh and solution of the linear elasticity problem (17) on the cylinder for $\mu_{+} / \mu_{-} \neq 1$. The solution has a kink along the interface and error concentrates at the side of the interface outside the cylinder.

Table 1 summarizes the main parameters and computational strategies used in the numerical examples. The variety of complex shapes and benchmarks considered above are intended to exhibit the good behaviour of interface $\mathrm{AgFEM}$, in as many situations as possible. Our numerical tests consider first numerical verification of the theoretical results proved in Section 3.2. In Section 4.3, we carry out convergence tests in uniform and $h$-adaptive meshes to show that interface AgFEM recovers optimal convergence rates. Afterwards, we examine, in Section 4.4, robustness w.r.t. cut location and material contrast, by means of geometry and material perturbations. We show that the condition number of the linear system, after diagonal scaling, is independent of cut location and material contrast. Finally, in Section 4.5, we assess good parallel performance and scalability with a weak-scaling analysis of some selected cases from the convergence tests. For each type of numerical test, we perform a subset of the possible matrix of cases in Table 1. We provide details for each subset, when dealing with the corresponding test. But before all that, we inform next about the computational infrastructure and software employed.

4.2. Experimental environment. Serial experiments are launched at the TITANI cluster of the Universitat Politècnica de Catalunya (Barcelona, Spain), whereas parallel experiments are carried out at the Marenostrum-IV (MN-IV) supercomputer, hosted by the Barcelona Supercomputing Centre. A message passing interface (MPI) parallel implementation of the interface $h$-AgFEM method is available at FEMPAR [56]. FEMPAR is linked against p4est v2.2 [57], as the octree Cartesian grid manipulation engine, and PETSc v3.11.1 [73] distributed-memory linear algebra data structures and solvers. Besides, condition number estimates are computed outside FEMPAR with MATLAB function condest. $^{3}$

Concerning linear solvers, a sparse direct solver from the MKL PARDISO package [74] is employed for serial tests. In contrast, a preconditioned conjugate gradient (CG) method is adopted for parallel tests. The selected preconditioner is a smoothed-aggregation algebraic multigrid (AMG) scheme called GAMG [72]. The linear solver is set up as in [54], with the aim of reducing, as much as possible, deviation from the default configuration given by GAMG. In order to advance the convergence test down to low global energy-norm error values, without being polluted by linear solver accuracy, convergence of GAMG is declared when $\|\mathbf{r}\|_{2} /\|\mathbf{b}\|_{2}<10^{-9}$ within the first 500 iterations, where $\mathbf{r} \doteq \mathbf{b}-\mathbf{A} \mathbf{x}^{\mathrm{cg}}$ is the unpreconditioned residual. Both (serial and parallel) solvers and preconditioner are readily available through the Krylov Methods KSP module of PETSc.

\footnotetext{
${ }^{3}$ MATLAB is a trademark of THE MATHWORKS INC.
} 


\begin{tabular}{ll}
\hline Description & Considered methods/values \\
\hline Model problem & interface Poisson, interface linear elasticity \\
Problem geometry & 2D: circle, flower, pacman shape; 3D: cylinder, \\
& popcorn flake, spiral, popcorn pacman, gyroid \\
out-FE-space (14), Fichera corner (15), & single shock (16), cylindrical inclusion (17) \\
Benchmark & serial and parallel \\
Experimental computer environment & p4est library [57] \\
Parallel mesh generation and partitioning tool & single quadtree (2D) or octree (3D) \\
Mesh topology & uniform, $h$-adaptive with Li and Bettess [67] criterion \\
Remeshing strategy & $\eta_{0}=0.25$ \\
Well-posed cut cell criterion & AgFE and StdFE \\
FE spaces & Q1 and Q2 hexahedral cells \\
Cell type and FE interpolation & sparse direct (serial) \\
Linear solver & preconditioned conjugate gradients (parallel) \\
Parallel preconditioner & smoothed-aggregation GAMG [72] \\
GAMG stopping criterion & $\|\mathbf{r}\|_{2} /\|\mathbf{b}\|_{2}<10^{-9}$ \\
Weights in averaged normal fluxes & $w^{+}=\frac{k^{-}}{k^{+}+k^{-}}$and $w^{-}=\frac{k^{+}}{k^{+}+k^{-}}$(Poisson) \\
& $w^{+}=\frac{\mu^{-}}{\mu_{+}+\mu_{-}}$and $w^{-}=\frac{\mu^{+}}{\mu_{+}+\mu_{-}}$(elasticity) \\
Coef. in Nitsche's penalty term for AgFEM & $\beta=10.0 q^{2}, q$ is the FE interpolation order \\
\hline
\end{tabular}

TABLE 1. Summary of main parameters and computational strategies used in the numerical examples

4.3. Convergence tests. We study the convergence of interface AgFEM in two stages. In the first one, we choose benchmark (14) and examine the rate at which the relative energy norm error decays with uniform mesh refinements. In a second stage, we consider the remaining benchmarks and observe the behaviour for both uniform and error-driven $h$-adaptive mesh refinements. All experiments run on five MN-IV nodes, i.e. we use a total of 240 CPUs, with each CPU mapped to a different MPI task.

For the first part, we consider the circle, flower, popcorn and spiral interface geometries. In the first three cases, the level sets are centred at the origin of coordinates and the physical domain is the unit $[0,1]^{3}$ cube, while the physical domain of the spiral case is the $[-1,1]^{2} \times[0,2]$ cuboid. In all cases, the interface cuts the external boundary. Besides, the circle has radius 0.7 and the flower level-set function in polar coordinates is $\varphi(r, \theta)=r-0.7(1+0.3 \sin (5 \theta))$. We refer to $[17,51]$ for the remaining level-set function expressions. The cuboid is initially meshed with a uniform Cartesian grid. Figure 7 gathers all convergence tests on uniform meshes for problem (14). In agreement to Proposition 3.5, we observe that AgFEM consistently recovers optimal convergence rates in the $H^{1}$-seminorm (equivalent to the energy norm) for all cases considered, including first and second order interpolations and extreme material contrasts.

For tests with uniform and $h$-adaptive mesh refinements, we consider (a) the Fichera-corner (15) on the pacman (2D) and popcorn-pacman (3D) shapes, (b) the single-shock (16) on the gyroid and (c) the cylindrical inclusion (17) on a cylinder. The physical domains are $[0,1]^{d},[-2,2]^{3}$ and $[0,1]^{3}$, resp. Geometry and numerical solutions for each case are represented in Figures 5, 4F and 6. We note that, in (a) the interface is in the interior of $\Omega$, while in (c) we exploit radial symmetry. We recall that the AMR process is driven by computing the exact discretisation error and the Li and Bettess convergence criterion [67], see Section 4.1. As shown in Figure 8, optimal convergence rates are retained both with uniform and $h$-adaptive mesh refinements, regardless of extreme material contrast values and order of approximation. Let us further justify this result:

Even though the solution to the Fichera-corner does not depend on material parameters, convergence rates do. In the Fichera case with uniform refinements, global error decreases at a rate of 2:3, when discrete error concentrates in $\Omega^{-}$, since $u^{-} \in H^{1+\frac{2}{3}}\left(\Omega^{-}\right)$has limited regularity. Conversely, standard convergence rates hold, when discrete error concentrates in $\Omega^{+}$, where $u^{+}$is smooth. Material contrast regulates which side of $\Gamma$ initially contributes more to numerical error, although when $h \rightarrow 0$ global error always converges at the slowest rate. We see that, for $k^{+} / k^{-}=1$, global error clearly concentrates in $\Omega^{-}$, while it concentrates in $\Omega^{+}$for $k^{+} / k^{-}=10^{6}$. For an intermediate value, e.g. $k^{+} / k^{-}=10^{3}$, discrete error initially concentrates in $\Omega^{+}$, but for $h$ small enough it shifts to $\Omega^{-}$. 


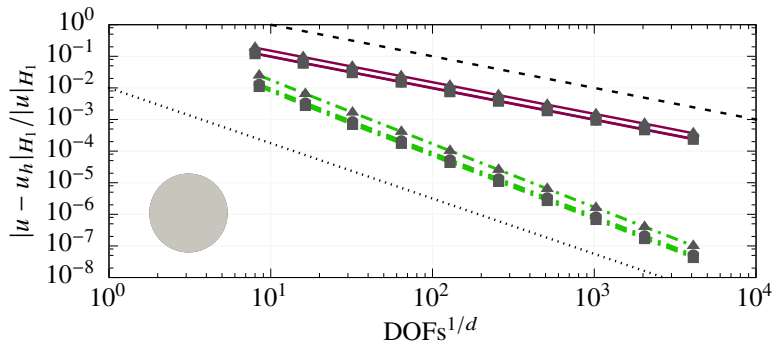

(A) Disk

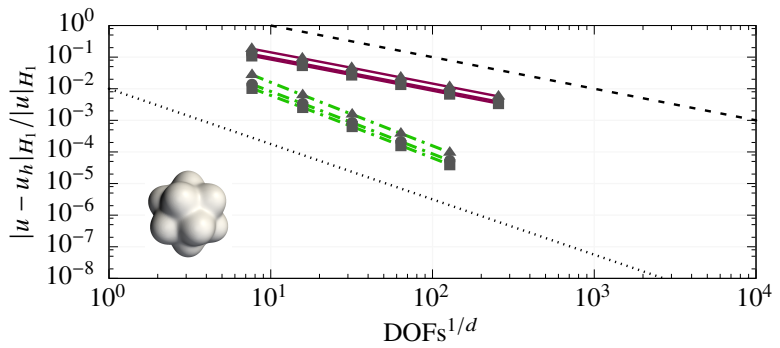

(c) Popcorn

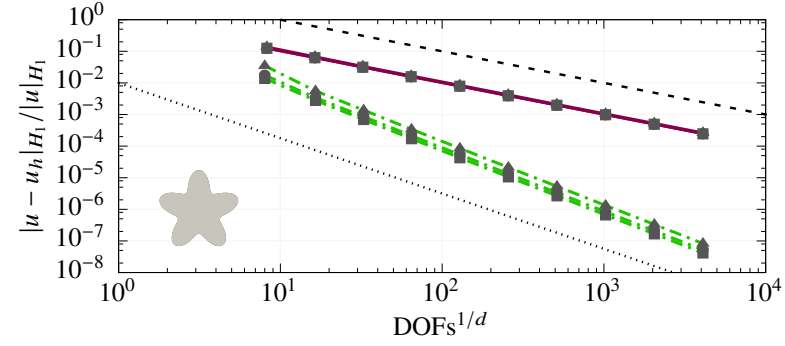

(в) Flower

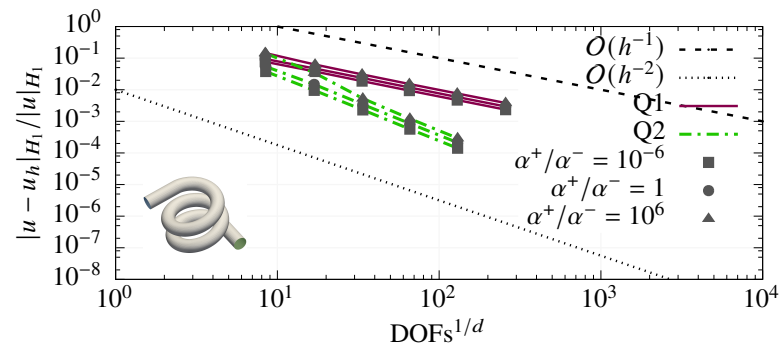

(D) Spiral

FIGURE 7. Convergence tests on uniform meshes: For benchmark (14) and an initial uniform mesh, AgFEM consistently shows optimal convergence rates as the mesh is uniformly refined.

As expected, $h$-adaptive refinements eliminate the influence of regularity of $u^{-}$on the convergence rates. However, as shown in Figure 5, different values of the material contrast produce different refinement patterns, in consistence with the discrete error distribution, as discussed above. In particular, mesh refinements concentrate in $\Omega^{-}$(or $\Omega^{+}$), when $k^{+} / k^{-}$is small (or large).

Since the single-shock case in the gyroid is rather intricate, convergence rates are initially suboptimal; optimal convergence rates are reached asymptotically (especially for quadratic FEs). We observe that, in front of uniform refinements, AMR is capable of entering faster into the asymptotic regime. However, the pace at which this is achieved depends on material contrast. In particular, larger values of $k^{+} / k^{-}$slow down reaching optimal rates.

Apart from that, results for the linear elasticity problem also deserve attention. We identify that the energy norm of the error decreases at a rate of 1:2 for linear FEs and 1:4 for quadratic FEs. This means we obtain superconvergence $\left(O\left(h^{q+1}\right)\right)$ for linear FEs and ultraconvergence $\left(O\left(h^{q+2}\right)\right)$ for quadratic FEs. Although we do not have conclusive evidence, we believe this behaviour is explained by the fact that Gauss-Legendre quadrature points on hexahedral cells are superconvergent stress recovery points [75]. In our case, when the cell is not intersected by $\Gamma$, local errors $\left\|(\boldsymbol{\sigma}: \boldsymbol{\varepsilon})\left(\boldsymbol{u}-\boldsymbol{u}_{h}\right)\right\|_{\boldsymbol{L}^{2}(\Omega)}$ are integrated with standard Gauss-Legendre quadrature rules. As a result, even though the approximated solution is not superconvergent, local error is computed at points that are superconvergent. In contrast, quadrature rules are locally modified in cut cells, as usual in unfitted FE methods [17]; thus, local errors in those cells are not computed at superconvergent points. In spite of this, it is clear from the convergence plots that the behaviour of the global error in the energy norm is not influenced by cut cells, i.e. global error retains the local superconvergence property that (only) holds in non-cut cells.

4.4. Robustness with respect to cut location and material contrast. For the sensitivity of AgFEM to cut location and material contrast, we restrict ourselves to the Poisson benchmark (14) in the flower and popcorn interfaces and the linear elasticity benchmark (17) in the cylinder.

Our approach is similar to the one in [36]. It consists in carrying out a batch of simulations in a biparametric space, considering different material contrast and cut configurations, as shown in Figure 9. The procedure is as follows. We start with a reference simulation in a unit cube $[0,1]^{d}$, that takes $k^{+} / k^{-}=1$ for $(14)$, or $\mu_{+} / \mu_{-}=1$ for (17). The unit cube is uniformly meshed with cell size $h=2^{-6+q}$ for (14), and $h=2^{-5+q}$ for (17), where $q$ is the FE interpolation order. The material perturbation simply consists in varying the material contrast $k^{+} / k^{-}$ or $\mu_{+} / \mu_{-}$of the reference simulation in the interval $\left[10^{-6}, 10^{6}\right]$. On the other hand, to produce different cut configurations, the unit cube is scaled to $[0,1+a h]^{d}$, where $a \in[-1,1]$. We remark that the number of mesh cells is kept constant, i.e. the cell size after scaling is $\hat{h}=(1+a h) h$.

Given this setting, we launch simulations with AgFEM for different pairs of $\left(k^{+} / k^{-}, a\right)$ or $\left(\mu_{+} / \mu_{-}, a\right)$, until we sweep the range $\left[10^{-6}, 10^{6}\right] \times[-1,1]$. We consider both serial and parallel computations; the latter are carried out in a single MN-IV node, i.e. 48 tasks. Along the sweep, we gather $H^{1}$-seminorm errors and condition number estimates. Afterwards, we condense the results into colour maps that plot the values each of 


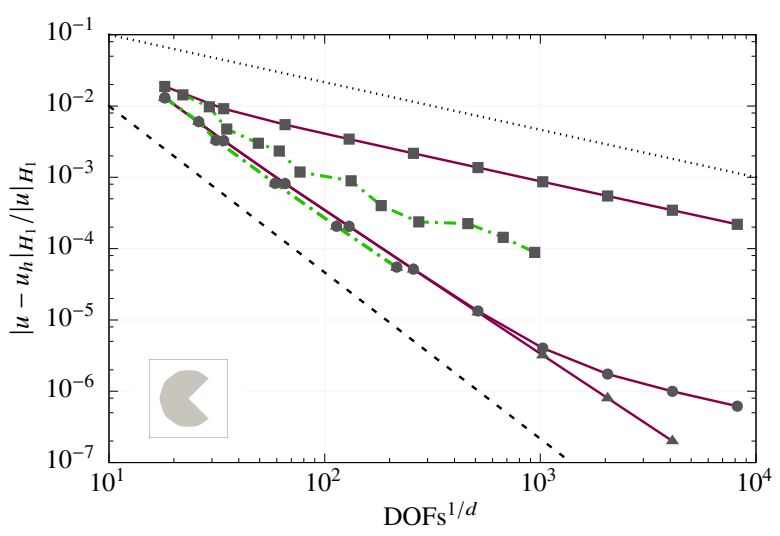

(A) Pacman-Fichera 2D

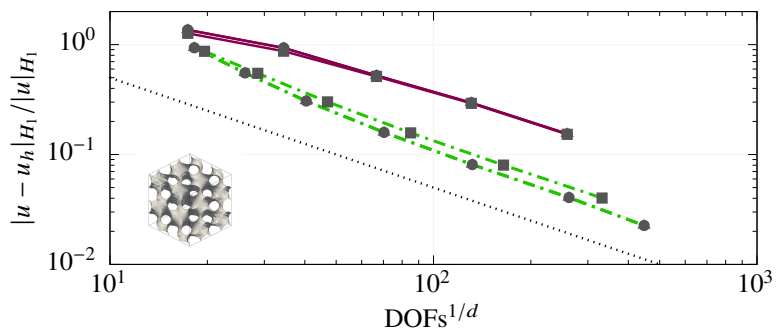

(c) Gyroid-shock linear FEs

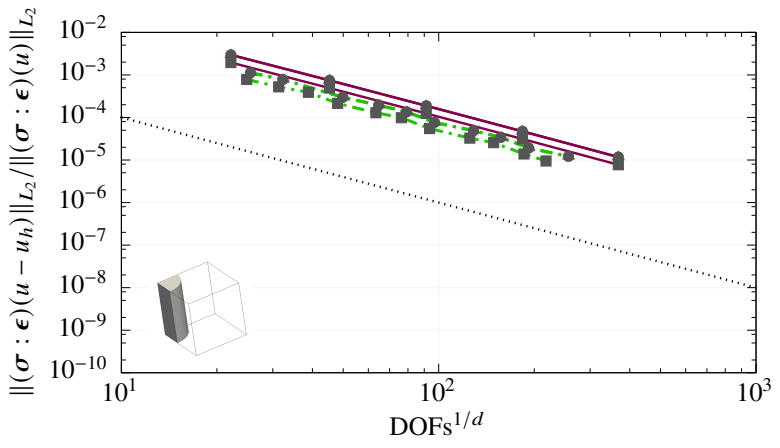

(E) Cylinder-elasticity linear FEs

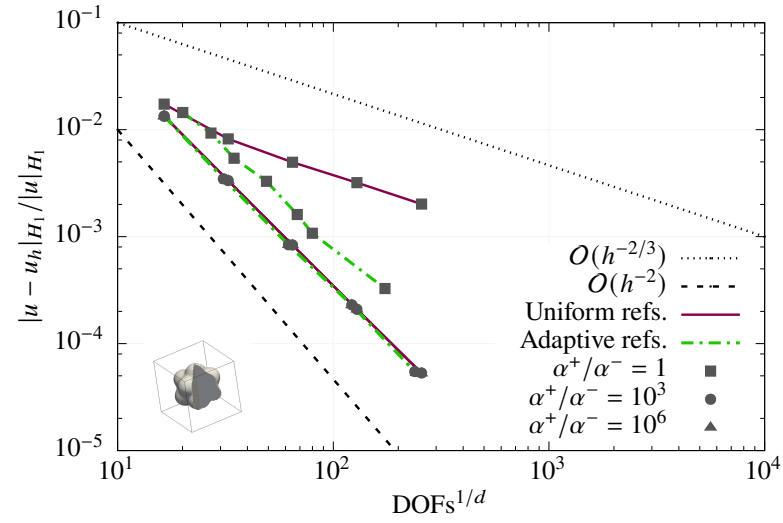

(в) Pacman-Fichera 3D

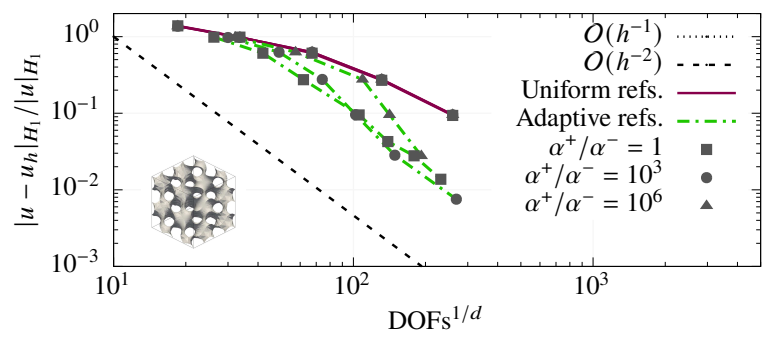

(D) Gyroid-shock quadratic FEs

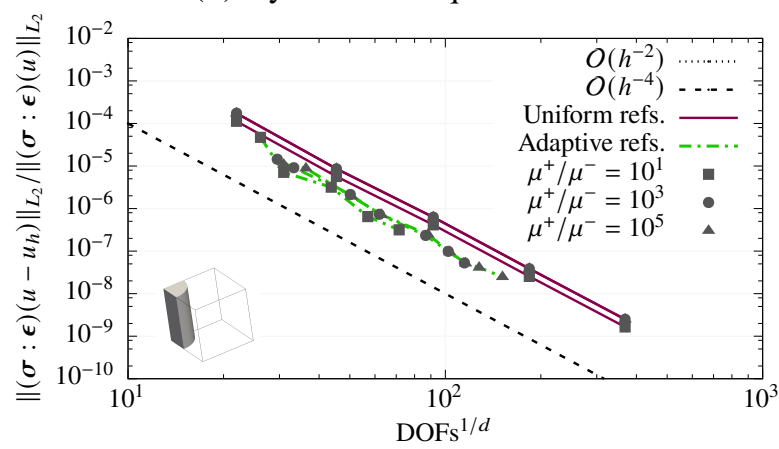

(F) Cylinder-elasticity quadratic FEs

FIgURE 8. Convergence tests on $h$-adaptive meshes: (a)-(b) h-adaptivity test with the Fichera-corner problem (15) for quadratic FEs: AgFEM reproduces the behaviour of standard FEM in body-fitted meshes, i.e. convergence rates with uniform refinements is limited by solution regularity, whereas optimal convergence rates are restored with AMR. (c)-(d) h-adaptivity test with the single-shock problem (16) on the gyroid: $h$-AgFEM holds (asymptotically) optimal convergence rates. (e)-(f) h-adaptivity test with the cylindrical inclusion problem (17) on the cylinder: energy norm error using $h$-AgFEM decays at optimal superconvergent rates.

these quantities in the $\left(k^{+} / k^{-}, a\right)$ or $\left(\mu_{+} / \mu_{-}, a\right)$ planes. We discuss next some of the results obtained with this procedure, represented in Figures 10, 11 and 12.

As seen in Figure 10, numerical errors in the $H^{1}$-seminorm are barely sensitive to material contrast and cut location. This behaviour is consistently observed in all three cases and linear/quadratic FEs. Although, for the linear elasticity case (17), the error decreases one order of magnitude around $\mu_{+} / \mu_{-}=1$, this is attributed to the fact that the solution is more regular when $\mu_{+} / \mu_{-}=1$ (it does not have a kink), not to the material contrast.

In Figure 11, we plot condition numbers obtained with one of the three cases, namely the Poisson equation (14) on the popcorn interface. We have additionally swept the parametric space with StdFE, for comparison with AgFE; it clearly illustrates the effect of the latter on the conditioning of the matrix. As shown in Figures 11A and $11 \mathrm{~B}$, the condition number of the linear system is extremely high for StdFE. While these large estimates are likely affected by a large numerical error, they clearly demonstrate the high sensitivity of StdFEM to the cut configuration. Besides, the problem can be so ill-conditioned that the local eigenvalue solver to compute $\beta$ breaks down. In contrast, AgFEM is fully robust and brings down condition numbers to values that the solvers can cope with, see Figures 11C and 11D. Besides, dependence on cut location vanishes completely, although there is a clear sensitivity to material contrast. Nonetheless, this dependence is not present in the condition number 


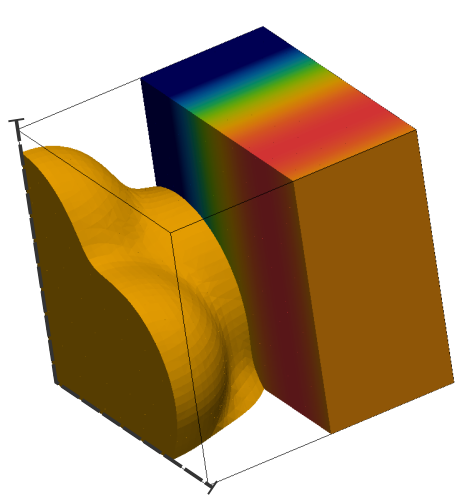

(A) $k^{+} / k^{-}=10^{-6}$ and $a=-1$

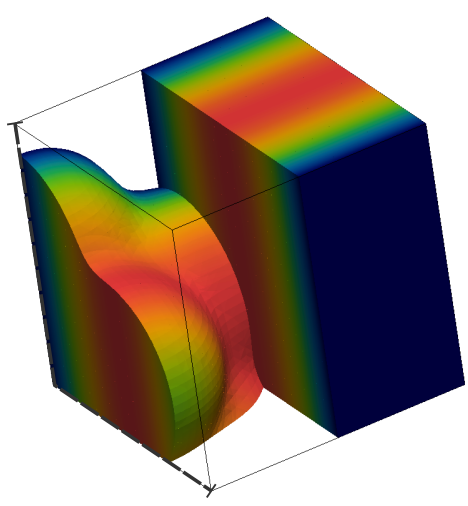

(в) $k^{+} / k^{-}=1$ and $a=0$

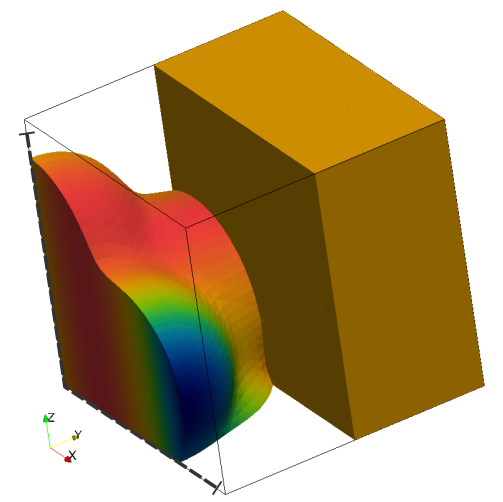

(c) $k^{+} / k^{-}=10^{6}$ and $a=1$

FIGURE 9. Illustration of the approach to study robustness w.r.t. cut location and material contrast on the popcorn interface. Note that we only show the right half of the subdomain outside the popcorn flake. To study sensitivity to material contrast, we vary $k^{+} / k^{-}$between $10^{-6}$ and $10^{6}$. To study sensitivity to cut location, we produce different cut locations by uniformly shrinking (Figure 9A) or stretching (Figure 9c) the physical domain with a parameter $a \in[-1,1]$ (dashed lines show the $x$ and $z$ dimensions of the cube represented in Figure 9в, as reference to compare the different cube scalings).

of the diagonally-scaled system matrix. Indeed, as seen in Figures $12 \mathrm{~A}$ and $12 \mathrm{~B}$, the condition number after diagonal scaling becomes barely sensitive to both cut location and material contrast. Furthermore, condition numbers are around $O\left(10^{4}\right)$, in the worst case, which is a rather low value for unfitted 3D+Q2 simulations. The same outcome is observed for the linear elasticity case, as shown in Figures 12C and 12D.

4.5. Weak-scaling analysis. We carry out weak-scaling tests for three $h$-adaptive cases studied in the convergence tests: (1) the Pacman-Fichera 3D with quadratic FEs for $k^{+} / k^{-}=1$ (Figure 8B) and the gyroid-shock with (2) linear (Figure 8C) and (3) quadratic (Figure 8D) FEs for $k^{+} / k^{-}=10^{3}$. In the analysis, we aim (a) to deploy a testing methodology that accounts for the fact that cells (and DOFs) that cut the interface are replicated and (b) to demonstrate that both the cell aggregation scheme and the set up of the AgFE space $\mathcal{V}_{h}$ are computationally (weakly) scalable. In the sequel we use $N_{\square}$ and $n_{\square}$ to denote global (i.e. referring to the whole mesh/domain) and local (i.e. referring to the processor-owned submesh/subdomain) sizes/cardinalities of a quantity $\square$.

Our strategy is analogous to the one detailed in [50]; it consists in repeating the convergence test, adjusting the number of processors to compute each point in the error plot. The goal is to impose that a suitable quantity remains (approximately) invariant across the whole convergence test. In addition, given a point, it is desirable that the invariant also holds across processors, in order to reduce noise in the results due to interprocessor imbalance. In FE simulations, the typical invariant is the (local) number of (free) DOFs each processor owns, since complexity of major phases (e.g. solving the linear system) depends on the number of DOFs. However, it is difficult to balance DOFs across processors in our meshes, which have both free and (hanging and ill-posed) constrained DOFs that overlap at the interface. For this reason, we choose as invariant the local number of active cells $n_{\mathrm{A} \text {,cells }}$, where the global counterpart is $N_{\mathrm{A}, \text { cells }}=N_{\mathcal{T}_{h, \mathrm{~A}}^{+}}+N_{\mathcal{T}_{h, \mathrm{~A}}^{-}}$, i.e. the number of cells in $\mathcal{T}_{h}$, but counting cells at the interface twice.

According to this, we consider the sequence of optimal AMR meshes, obtained in the convergence test, and compute the number of processors for the weak-scaling analysis as

$$
P^{i}=P^{1}\left\lfloor\frac{N_{\mathrm{A}, \text { cells }}^{i}}{N_{\mathrm{A}, \text { cells }}^{1}}\right\rfloor, i>1,
$$

where superscript $i>1$ refers to each element in the sequence of optimal meshes (points in the error curve), $P^{1}$ is a fixed initial number of processors and $\lfloor\cdot\rfloor$ is the floor function; given a real number $x,\lfloor x\rfloor$ is the greatest integer less than or equal to $x$. Table 2 gathers the sequences $\left\{P^{i}\right\}_{i>1}$ obtained following this procedure, for the three cases that are studied in this section. We observe that (1) it is clearly more straightforward to equally distribute active cells among processors than DOFs and (2) the (average) local number of free DOFs grows mildly with $i>1$. Hence, this approach allows us to (conservatively) examine how the problem scales with DOFs, avoiding cumbersome strategies to balance DOFs.

Once established the weak-scaling methodology, our purpose is to show that remarkable scalability of ( $h$ adaptive) AgFEM, reported in previous works for problems with unfitted boundary [50, 54], is preserved for interface problems. As those works have already addressed weak scalability of the whole FE simulation 


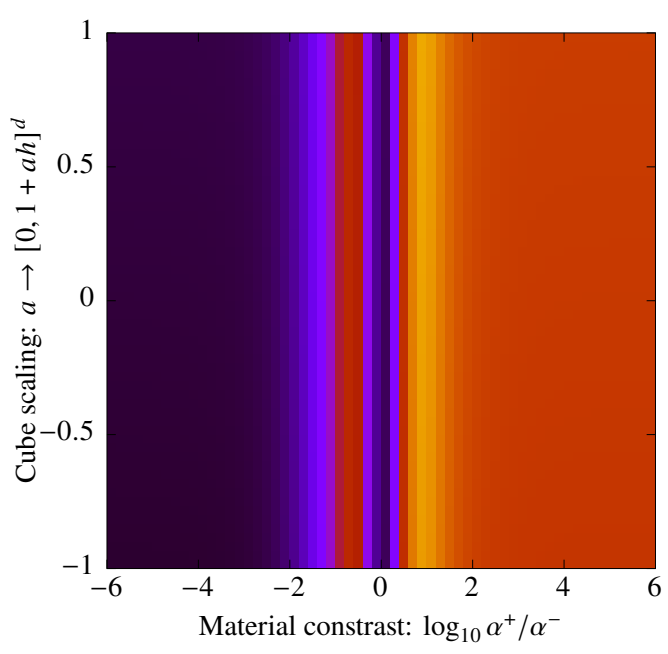

(A) Flower and (14) with Q1 FEs.

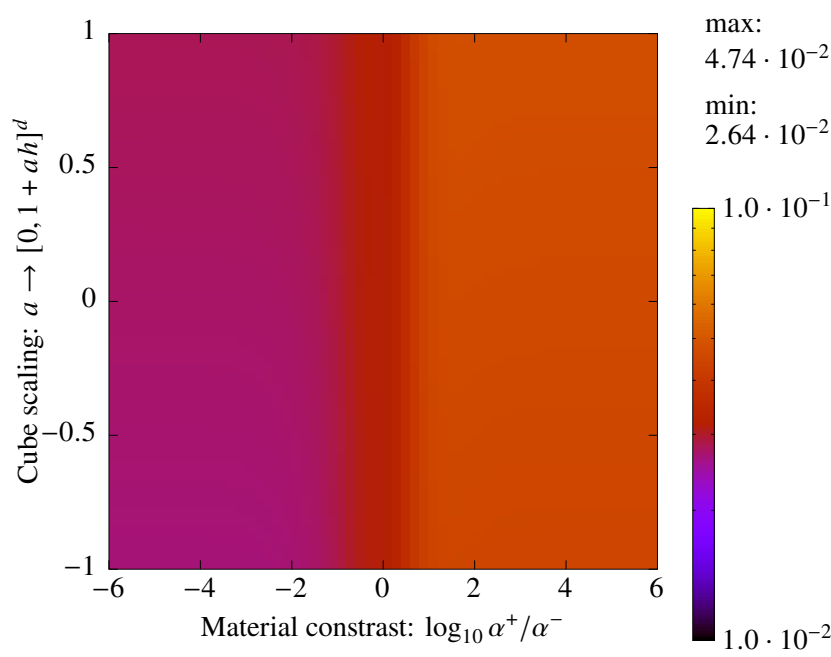

(c) Popcorn and (14) with Q1 FEs.

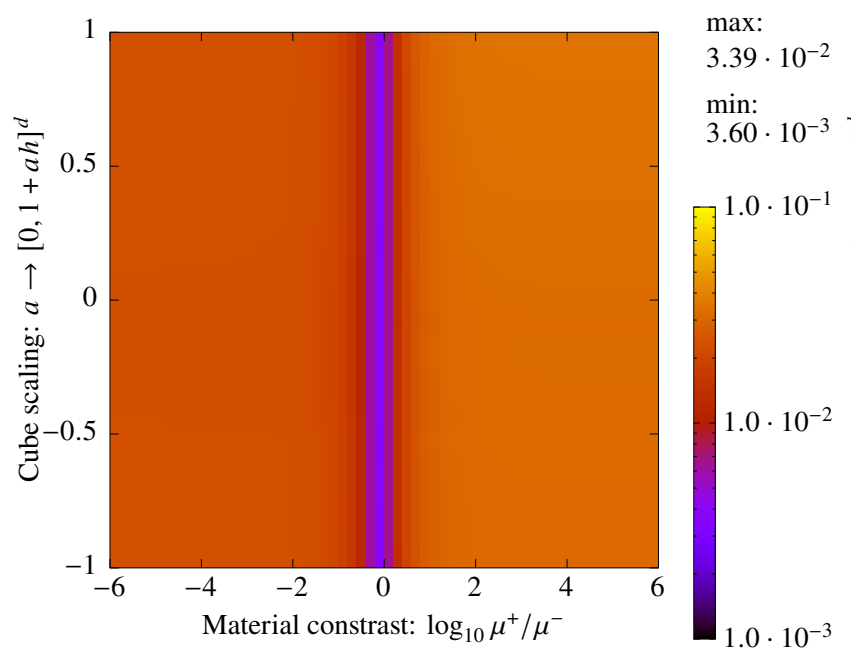

(E) Cylinder and (17) for Q1.

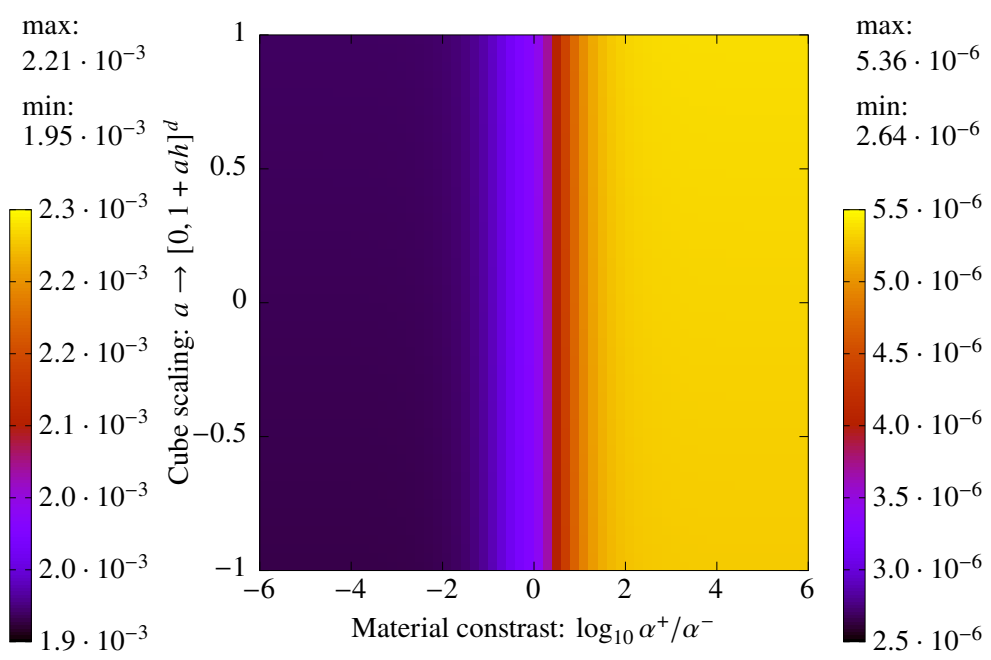

(в) Flower and (14) with Q2 FEs.

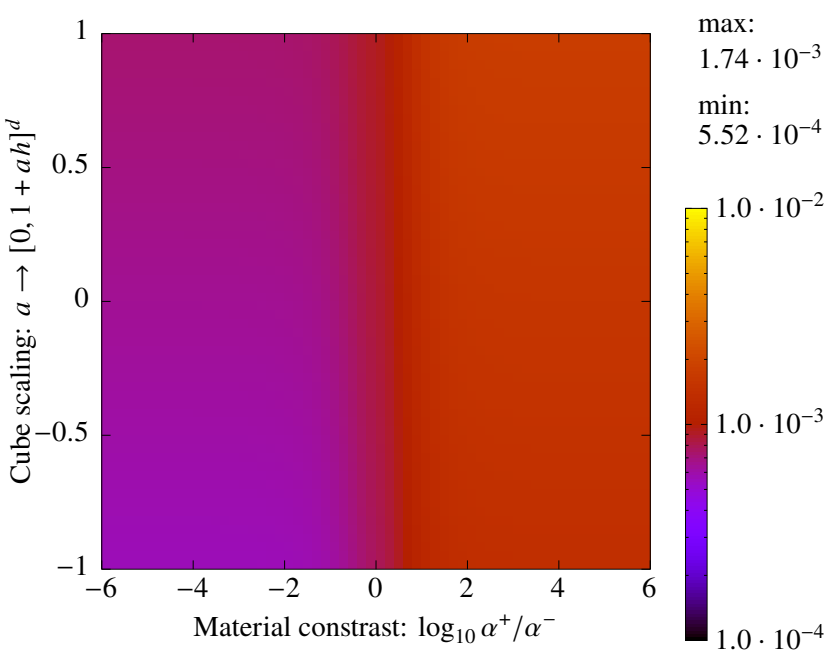

(D) Popcorn and (14) with Q2 FEs.

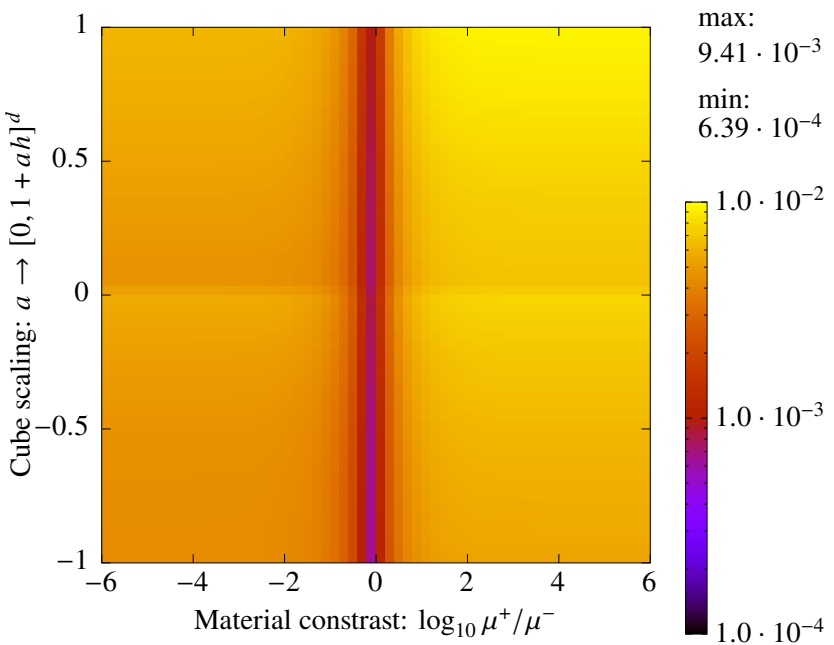

(F) Cylinder and (17) for Q2.

FIGURE 10. Sensitivity test of Ag FEM w.r.t. material contrast and cut location: For the cases described in Section 4.4, the $H^{1}$-seminorm relative error, i.e. $\left|u-u_{h}\right|_{H^{1}} /|u|_{H^{1}}$, is barely sensitive to material contrast and cut location.

pipeline, we focus on reporting wall clock times spent in the two main AgFEM-specific phases, i.e. those phases particular of our approach, not present in other unfitted techniques. The two phases are (1) cell aggregation, 


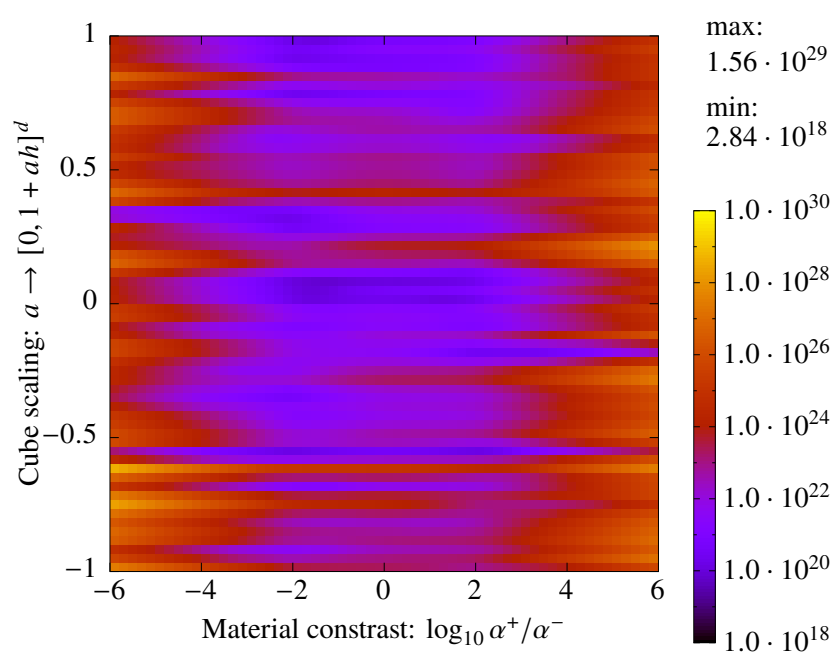

(A) Standard FEM with Q1.

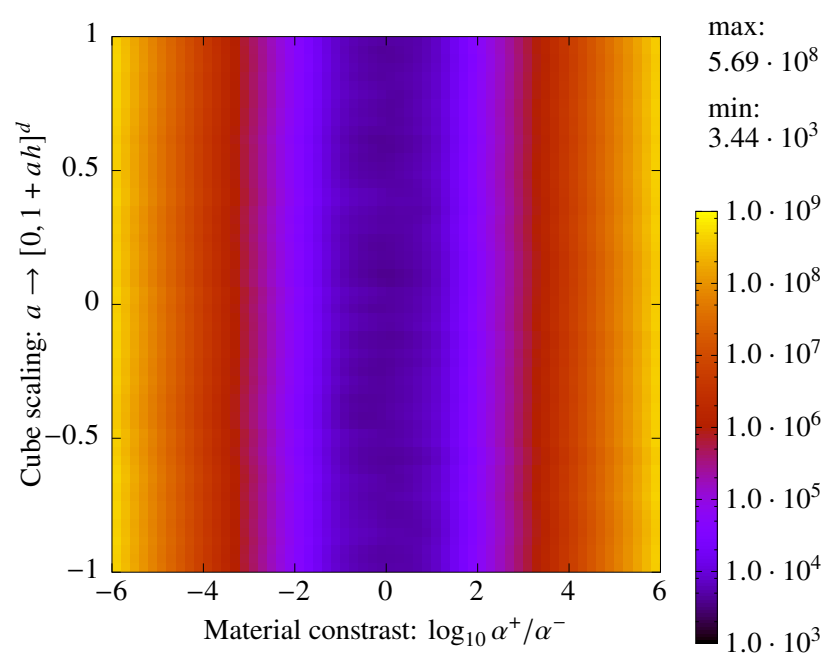

(c) AgFEM with Q1.

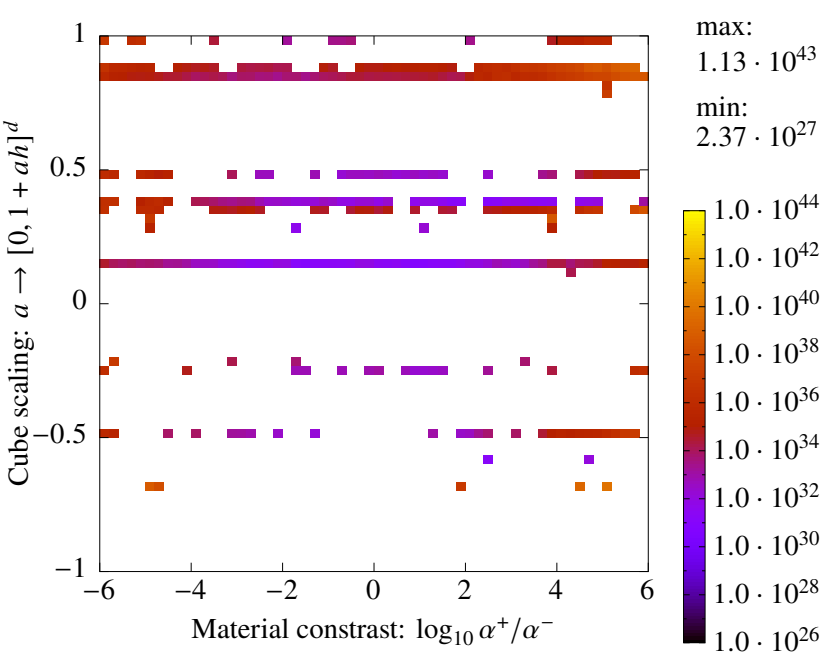

(в) Standard FEM with Q2.

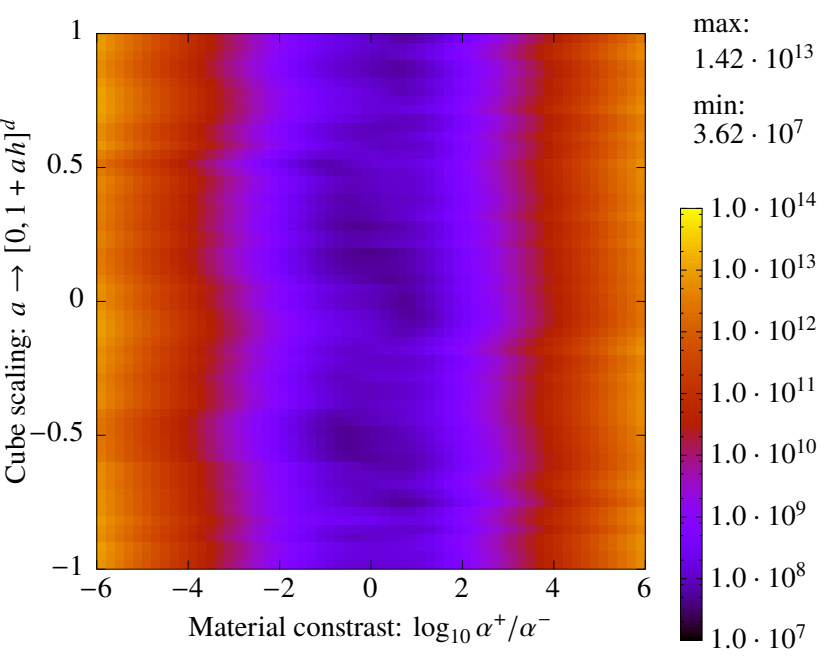

(D) AgFEM with Q2.

FIGURE 11. Sensitivity test w.r.t. material contrast and cut location for popcorn example. Examination of condest $(A)$ exposes how lack of robustness and dependency on cut location in standard FEM is not present in AgFEM.

see Section 2.2, and (2) setup of the AgFE space, see Section 2. As finding the optimal mesh for each $i>1$ is an iterative AMR process, we only monitor these quantities for the optimal mesh (last iteration). We note that, even though (1) and (2) are critical phases of the simulation, from the computational viewpoint, they are not the most prominent ones. Thus, AgFEM does not affect much overall run time with respect to a standard (ill-posed) Galerkin method.

To allocate the MPI tasks in the MN-IV supercomputer, we resort to the default task placement policy of Intel MPI (v2018.4.057) with partially filled nodes. For each point of the test, the number of nodes $N^{i}$ is selected as $N^{i}=\left\lceil P^{i} / 48\right\rceil$, where $\lceil\cdot\rceil$ is the ceiling function; given a real number $x,\lceil x\rceil$ is the smallest integer more than or equal to $x$. If $P^{i}$ is not multiple of 48, the placement policy fully populates the first $N-1$ nodes with 48 MPI tasks per node; the remaining $P^{i}-48(N-1)$ MPI tasks are mapped to the last node.

Figure 13 gathers all the quantities surveyed in weak scaling tests. The main phases of $h$-adaptive AgFEM exhibit remarkable scalability for the three cases considered. We observe that the number of local active cells $n_{\mathrm{A}, \text { cells }}$ and DOFs $n_{\mathrm{dofs}}^{i}, i>1$ for the gyroid-shock AMR-Q1 case are significantly larger than for the other two cases. That is why this case yields the largest computational times.

\section{Conclusions}

This work addressed a novel $h$-adaptive aggregated FE method for large-scale (unfitted) interface elliptic boundary value problems. Our methodology is grounded on the well-established approach of weakly coupling 

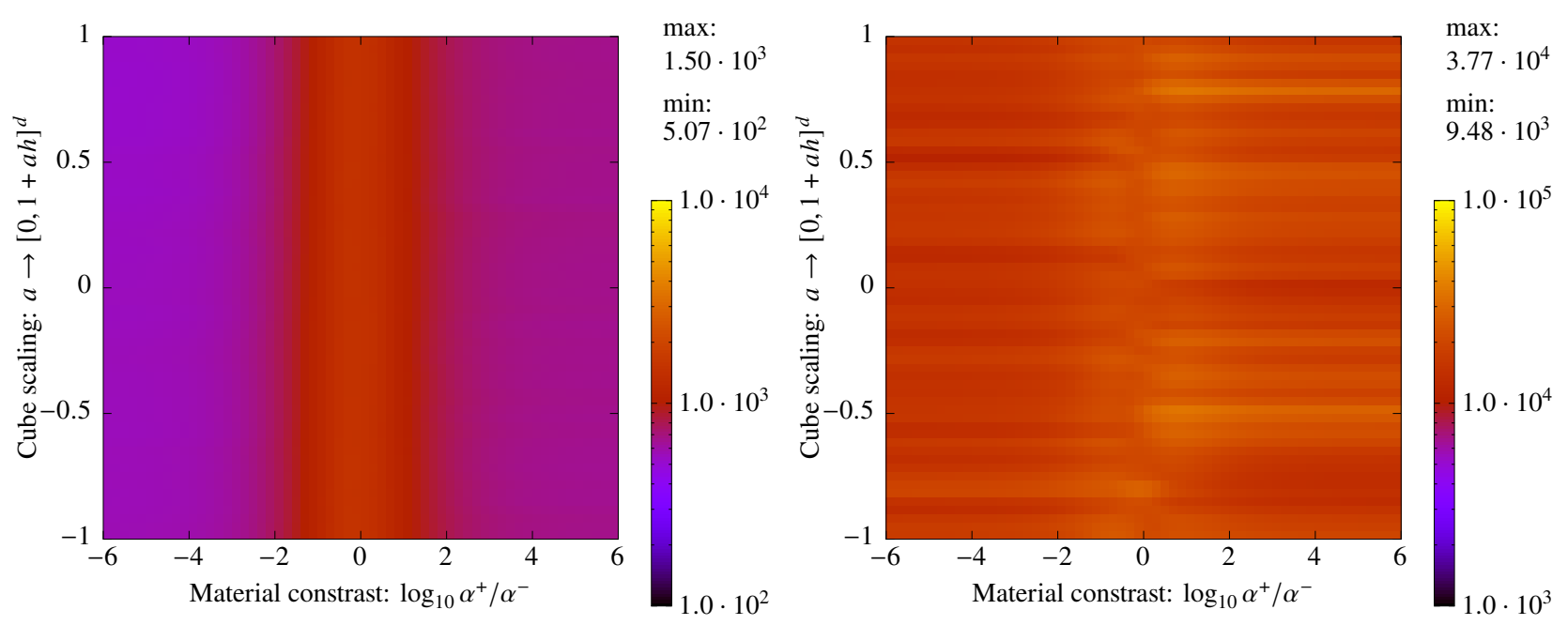

(A) Popcorn example (14): condest $\left(D^{-1} A\right)$ for Q1.

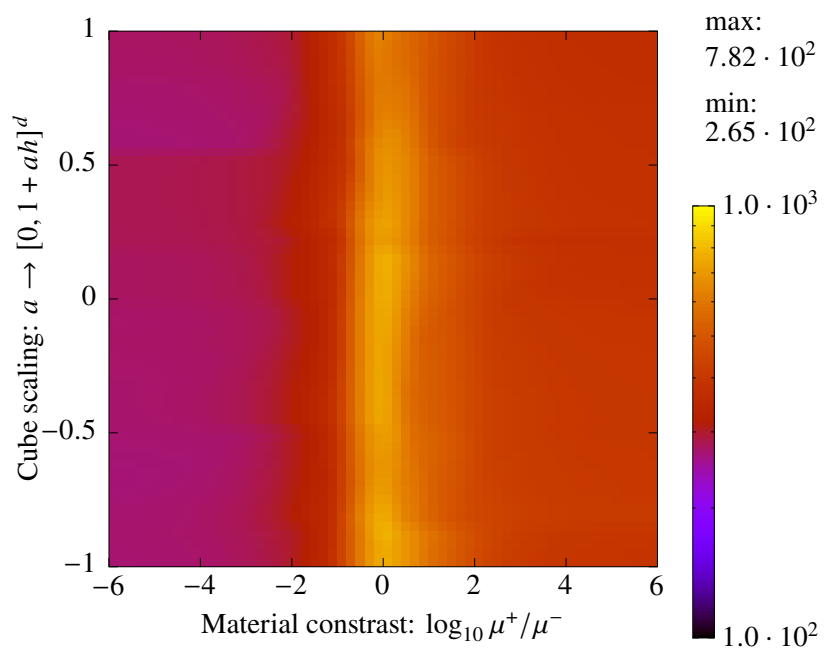

(в) Popcorn example (14): condest $\left(D^{-1} A\right)$ for Q2.

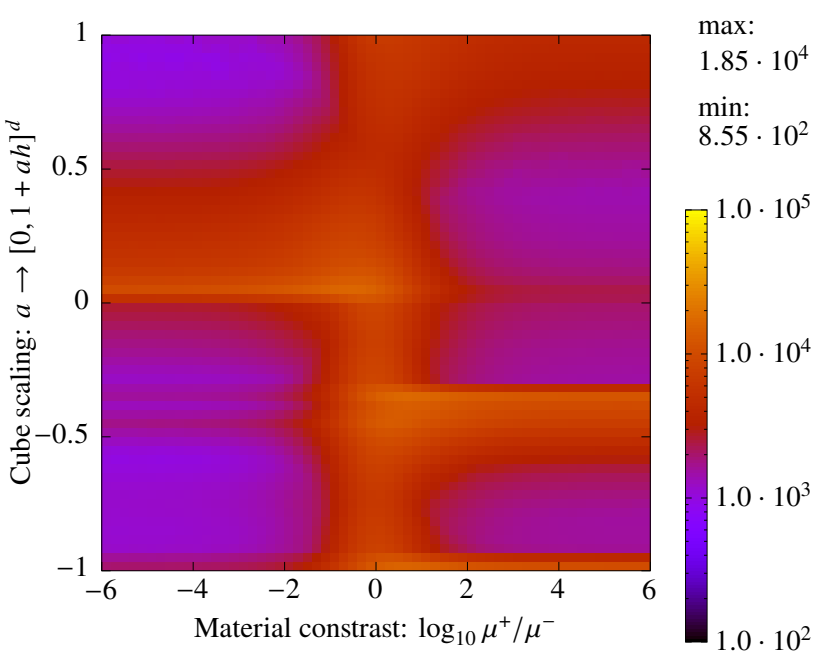

(c) Cylinder example (17): condest $\left(D^{-1} A\right)$ for Q1.

(D) Cylinder example (17): condest $\left(D^{-1} A\right)$ for $\mathrm{Q} 2$.

FIGURE 12. In AgFEM, condition number of the diagonally-scaled system matrix, i.e. $\operatorname{condest}\left(D^{-1} A\right)$, does not depend on cut location or material contrast and is effectively controlled; all condition numbers are down to $\mathcal{O}\left(10^{4}\right)$, in the worst case.

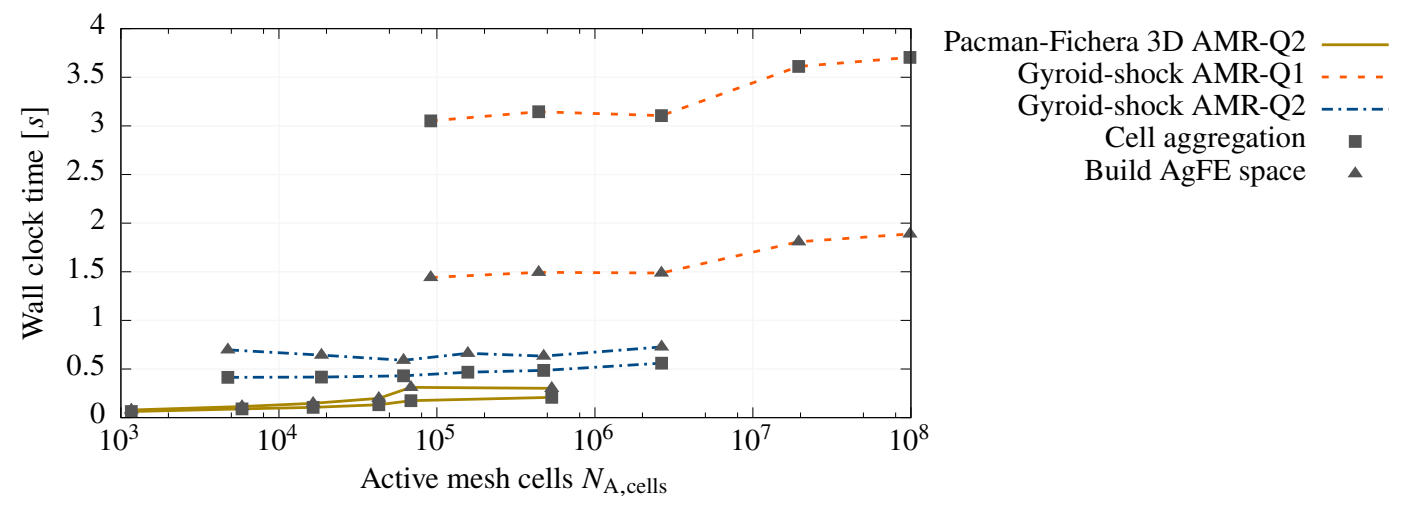

FIGURE 13. Weak scaling tests on selected interface problems from convergence tests in Section 4.3 up to 2,150 MPI tasks, as reported in Table 2 .

interface-overlapping discretisations [28] and the recently developed $h$-adaptive AgFE method [50] for unfitted boundary elliptic problems. The study of the new method is accompanied with complete theoretical characterisation and thorough numerical experimentation on a suite of Poisson and linear elasticity $(h p$-FEM) benchmarks with complex interface shapes. 


\begin{tabular}{|c|c|c|c|c|c|c|}
\hline \multicolumn{7}{|c|}{ Pacman-Fichera 3D AMR-Q2 and $n_{\mathrm{A}, \text { cells }} \approx 1.2 k$} \\
\hline$P$ & 1 & 5 & 14 & 36 & 58 & 457 \\
\hline$N_{\mathrm{A}, \text { cells }}$ & $1.2 \mathrm{k}$ & $5.8 \mathrm{k}$ & $16 \mathrm{k}$ & $43 \mathrm{k}$ & $67 \mathrm{k}$ & $533 \mathrm{k}$ \\
\hline$N_{\text {dofs }}$ & $8.0 \mathrm{k}$ & $42 \mathrm{k}$ & $118 \mathrm{k}$ & $315 \mathrm{k}$ & $510 \mathrm{k}$ & $4,031 \mathrm{k}$ \\
\hline$n_{\text {dofs }}$ & $8.0 \mathrm{k}$ & $8.3 \mathrm{k}$ & $8.3 \mathrm{k}$ & $8.6 \mathrm{k}$ & $8.6 \mathrm{k}$ & $8.8 \mathrm{k}$ \\
\hline \multicolumn{7}{|c|}{ Gyroid-shock AMR-Q1 and $n_{\mathrm{A}, \text { cells }} \approx 46 k$} \\
\hline$P$ & 2 & 9 & 57 & 556 & 2,150 & \\
\hline$N_{\mathrm{A}, \text { cells }}$ & $92 \mathrm{k}$ & $440 \mathrm{k}$ & $2,637 \mathrm{k}$ & $19,471 \mathrm{k}$ & $98,516 \mathrm{k}$ & \\
\hline$N_{\text {dofs }}$ & $66 \mathrm{k}$ & $348 \mathrm{k}$ & $2,288 \mathrm{k}$ & $18,056 \mathrm{k}$ & $89,822 \mathrm{k}$ & \\
\hline$n_{\mathrm{dofs}}$ & $33 \mathrm{k}$ & $36 \mathrm{k}$ & $40 \mathrm{k}$ & $42 \mathrm{k}$ & $42 \mathrm{k}$ & \\
\hline \multicolumn{7}{|c|}{ Gyroid-shock AMR-Q2 and $n_{\mathrm{A} \text {,cells }} \approx 4.7 k$} \\
\hline$P$ & 1 & 4 & 13 & 33 & 99 & 556 \\
\hline$N_{\mathrm{A}, \text { cells }}$ & $4.7 \mathrm{k}$ & $19 \mathrm{k}$ & $62 \mathrm{k}$ & $157 \mathrm{k}$ & $474 \mathrm{k}$ & $2,641 \mathrm{k}$ \\
\hline$N_{\text {dofs }}$ & $27 \mathrm{k}$ & $118 \mathrm{k}$ & $409 \mathrm{k}$ & $1,065 \mathrm{k}$ & $3,306 \mathrm{k}$ & $19,430 \mathrm{k}$ \\
\hline$n_{\mathrm{dofs}}$ & $27 \mathrm{k}$ & $30 \mathrm{k}$ & $32 \mathrm{k}$ & $32 \mathrm{k}$ & $33 k$ & $35 \mathrm{k}$ \\
\hline
\end{tabular}

TABLE 2. Number of subdomains $P$, global active cells $N_{\mathrm{A} \text {, cells }}$, global DOFs $N_{\mathrm{dofs}}$ and local DOFs $n_{\mathrm{dofs}}$ for the cases considered in the weak scaling tests of Figure 13. For each case, local active cells $n_{\mathrm{A}, \text { cells }}$, remains quasi-constant with $P$. Besides, $n_{\mathrm{dofs}}$ (slowly) increases monotonically.

As main contributions of the paper, we have introduced a (a) natural extension of the (distributed-memory) cell aggregation algorithm in [54] for $n$-interface problems. We have shown that (b) AgFE spaces easily blend to the typical Cartesian-product approximation structures of interface-overlapping meshes. We have proven (c) well-posedness and optimal approximation properties of a symmetric interior penalty method (SIPM)-AgFEM discrete formulation for the irreducible linear elasticity problem. Robustness w.r.t. cut location is ensured, by inheriting cut-independent estimates from AgFEM in unfitted boundaries, while robustness w.r.t. material contrast is achieved, by using the same weighted average of body-fitted DG methods. Besides, the resulting method admits (d) straightforward implementation on top of an existing large-scale implementation of AgFEM for unfitted boundary problems. To conclude, exhaustive numerical tests have exposed (e) optimal ( $h$-adaptive) approximation capability, robustness with respect to cut location and material contrast and remarkable scalability on parallel adaptive Cartesian tree-based meshes.

Our study offers compelling insight and evidence of the potential of AgFEM as an effective large-scale FE solver for complex multiphase and multiphysics problems modelled by PDEs. Extension to any of those problems is object of future work. Additionally, the paper provides useful guidance in applying other unfitted CG methods to interface problems, especially those relying on cell aggregation.

\section{ACKNOWLEDGEMENTS}

Financial support from the European Commission under the FET-HPC ExaQUte project (Grant agreement ID: 800898) within the Horizon 2020 Framework Programme is gratefully acknowledged. This work has been partially funded by the project MTM2014-60713-P from the "Ministerio de Economía, industria y Competitividad" of Spain. E. Neiva gratefully acknowledges the support received from the Catalan Government through a FI fellowship (2019 FI-B2-00090; 2018 FI-B100095; 2017 FI-B-00219). S. Badia gratefully acknowledges the support received from the Catalan Government through the ICREA Acadèmia Research Program. The authors thankfully acknowledge the computer resources at Marenostrum-IV and the technical support provided by the Barcelona Supercomputing Center (RES-ActivityIDs: IM-2019-3-0008, IM2020-1-0002). Financial support to CIMNE via the CERCA Programme / Generalitat de Catalunya is also acknowledged.

\section{REFERENCES}

[1] N. Sukumar, N. Moës, B. Moran, and T. Belytschko. Extended finite element method for three-dimensional crack modelling. International Journal for Numerical Methods in Engineering, 48(11):1549-1570, 2000. doi:10.1002/10970207(20000820)48:11<1549::AID-NME955>3.0.CO;2-A.

[2] H. Waisman and L. Berger-Vergiat. An adaptive domain decomposition preconditioner for crack propagation problems modeled by XFEM. International Journal for Multiscale Computational Engineering, 11(6):633-654, 2013. doi:10.1615/IntJMultCompEng.2013006012.

[3] L. Berger-Vergiat, H. Waisman, B. Hiriyur, R. Tuminaro, and D. Keyes. Inexact Schwarz-algebraic multigrid preconditioners for crack problems modeled by extended finite element methods. International Journal for Numerical Methods in Engineering, 90(3):311-328, 2012. doi:10.1002/nme.3318. 
[4] B. Schott, C. Ager, and W. A. Wall. Monolithic cut finite element-based approaches for fluid-structure interaction. International Journal for Numerical Methods in Engineering, 119(8):757-796, 2019. doi:10.1002/nme.6072.

[5] F. Alauzet, B. Fabrèges, M. A. Fernández, and M. Landajuela. Nitsche-XFEM for the coupling of an incompressible fluid with immersed thin-walled structures. Computer Methods in Applied Mechanics and Engineering, 301:300-335, 2016. doi:10.1016/j.cma.2015.12.015.

[6] S. Zonca, C. Vergara, and L. Formaggia. An unfitted formulation for the interaction of an incompressible fluid with a thick structure via an XFEM/DG approach. SIAM Journal on Scientific Computing, 40(1):B59-B84, 2018. doi:10.1137/16M1097602.

[7] A. Massing, M. G. Larson, A. Logg, and M. E. Rognes. A Nitsche-based cut finite element method for a fluidstructure interaction problem. Communications in Applied Mathematics and Computational Science, 10(2):97-120, 2015. doi:10.2140/camcos.2015.10.97.

[8] H. Sauerland and T. P. Fries. The extended finite element method for two-phase and free-surface flows: A systematic study. Journal of Computational Physics, 230(9):3369-3390, 2011. doi:10.1016/j.jcp.2011.01.033.

[9] R. Saye. Implicit mesh discontinuous Galerkin methods and interfacial gauge methods for high-order accurate interface dynamics, with applications to surface tension dynamics, rigid body fluid-structure interaction, and free surface flow: Part II. Journal of Computational Physics, 344:683-723, 2017. doi:10.1016/j.jcp.2017.05.003.

[10] M. Kirchhart, S. Gross, and A. Reusken. Analysis of an XFEM discretization for Stokes interface problems. SIAM Journal on Scientific Computing, 38(2):A1019-A1043, 2016. doi:10.1137/15M1011779.

[11] E. Burman, D. Elfverson, P. Hansbo, M. G. Larson, and K. Larsson. Shape optimization using the cut finite element method. Computer Methods in Applied Mechanics and Engineering, 328:242-261, 2018. doi:10.1016/j.cma.2017.09.005.

[12] F. Feppon, G. Allaire, F. Bordeu, J. Cortial, and C. Dapogny. Shape optimization of a coupled thermal fluid-structure problem in a level set mesh evolution framework. SeMA Journal, 76(3):413-458, 2019. doi:10.1007/s40324-01800185-4.

[13] E. Neiva, M. Chiumenti, M. Cervera, E. Salsi, G. Piscopo, S. Badia, A. F. Martín, Z. Chen, C. Lee, and C. Davies. Numerical modelling of heat transfer and experimental validation in powder-bed fusion with the virtual domain approximation. Finite Elements in Analysis and Design, 168:103343, 2020. doi:10.1016/j.finel.2019.103343.

[14] M. Carraturo, J. Jomo, S. Kollmannsberger, A. Reali, F. Auricchio, and E. Rank. Modeling and experimental validation of an immersed thermo-mechanical part-scale analysis for laser powder bed fusion processes. Additive Manufacturing, 36:101498, 2020. doi:10.1016/j.addma.2020.101498.

[15] S. Badia, J. Hampton, and J. Principe. Embedded multilevel monte carlo for uncertainty quantification in random domains. International Journal for Uncertainty Quantification, in press. doi:10.1615/Int.J.UncertaintyQuantification.2021032984.

[16] T. Belytschko, N. Moës, S. Usui, and C. Parimi. Arbitrary discontinuities in finite elements. International Journal for Numerical Methods in Engineering, 50(4):993-1013, 2001. doi:10.1002/1097-0207(20010210)50:4<993::AIDNME164>3.0.CO;2-M.

[17] E. Burman, S. Claus, P. Hansbo, M. G. Larson, and A. Massing. CutFEM: Discretizing Geometry and Partial Differential Equations. International Journal for Numerical Methods in Engineering, 104(7):472-501, 2015. doi:10.1002/nme.4823.

[18] D. Elfverson, M. G. Larson, and K. Larsson. CutIGA with basis function removal. Advanced Modeling and Simulation in Engineering Sciences, 5(1):6, 2018. doi:10.1186/s40323-018-0099-2.

[19] R. Mittal and G. Iaccarino. Immersed Boundary Methods. Annual Review of Fluid Mechanics, 37(1):239-261, 2005. doi:10.1146/annurev.fluid.37.061903.175743.

[20] D. Schillinger and M. Ruess. The Finite Cell Method: A review in the context of higher-order structural analysis of CAD and image-based geometric models. Archives of Computational Methods in Engineering, 22(3):391-455, 2015. doi:10.1007/s11831-014-9115-y.

[21] A. Main and G. Scovazzi. The Shifted Boundary Method for embedded domain computations. Part I: Poisson and Stokes problems. Journal of Computational Physics, 372:972-995, 2018. doi:10.1016/j.jcp.2017.10.026.

[22] D. Kamensky, M.-C. Hsu, D. Schillinger, J. A. Evans, A. Aggarwal, Y. Bazilevs, M. S. Sacks, and T. J. Hughes. An immersogeometric variational framework for fluid-structure interaction: Application to bioprosthetic heart valves. Computer methods in applied mechanics and engineering, 284:1005-1053, 2015. doi:10.1016/j.cma.2014.10.040.

[23] R. Saye. Implicit mesh discontinuous Galerkin methods and interfacial gauge methods for high-order accurate interface dynamics, with applications to surface tension dynamics, rigid body fluid-structure interaction, and free surface flow: Part I. Journal of Computational Physics, 344:647-682, 2017. doi:10.1016/j.jcp.2017.04.076.

[24] C. Engwer and F. Heimann. Dune-UDG: a cut-cell framework for unfitted discontinuous Galerkin methods. In Advances in DUNE, pages 89-100. Springer, 2012. doi:10.1007/978-3-642-28589-9_7.

[25] A. Johansson and M. G. Larson. A high order discontinuous Galerkin Nitsche method for elliptic problems with fictitious boundary. Numerische Mathematik, 123(4):607-628, 2013. doi:10.1007/s00211-012-0497-1.

[26] B. Müller, S. Krämer-Eis, F. Kummer, and M. Oberlack. A high-order discontinuous Galerkin method for compressible flows with immersed boundaries. International Journal for Numerical Methods in Engineering, 110(1):3-30, 2017. doi:10.1002/nme.5343. 
[27] W. E. H. Sollie, O. Bokhove, and J. J. van der Vegt. Space-time discontinuous Galerkin finite element method for two-fluid flows. Journal of computational physics, 230(3):789-817, 2011. doi:10.1016/j.jcp.2010.10.019.

[28] A. Hansbo and P. Hansbo. An unfitted finite element method, based on Nitsche's method, for elliptic interface problems. Computer methods in applied mechanics and engineering, 191(47-48):5537-5552, 2002. doi:10.1016/S00457825(02)00524-8.

[29] J. M. Melenk and I. Babuška. The partition of unity finite element method: basic theory and applications. In Research Report/Seminar für Angewandte Mathematik, volume 1996. Eidgenössische Technische Hochschule, Seminar für Angewandte Mathematik, 1996. doi:10.1016/S0045-7825(96)01087-0.

[30] É. Béchet, N. Moës, and B. Wohlmuth. A stable Lagrange multiplier space for stiff interface conditions within the extended finite element method. International Journal for Numerical Methods in Engineering, 78(8):931-954, 2009. doi:10.1002/nme.2515.

[31] E. Burman and P. Hansbo. Fictitious domain finite element methods using cut elements: I. A stabilized Lagrange multiplier method. Computer Methods in Applied Mechanics and Engineering, 199(41-44):2680-2686, 2010. doi:10.1016/j.cma.2010.05.011.

[32] P. M. Areias and T. Belytschko. A comment on the article "A finite element method for simulation of strong and weak discontinuities in solid mechanics" by A. Hansbo and P. Hansbo [Comput. Methods Appl. Mech. Engrg. 193 (2004) 3523-3540]. Computer methods in applied mechanics and engineering, 9(195):1275-1276, 2006. doi:10.1016/j.cma.2005.03.006.

[33] R. Stenberg. On some techniques for approximating boundary conditions in the finite element method. Journal of Computational and applied Mathematics, 63(1-3):139-148, 1995. doi:10.1016/0377-0427(95)00057-7.

[34] I. Babuška. The finite element method with penalty. Mathematics of computation, 27(122):221-228, 1973. doi:10.2307/2005611.

[35] J. Nitsche. Über ein Variationsprinzip zur Lösung von Dirichlet-Problemen bei Verwendung von Teilräumen, die keinen Randbedingungen unterworfen sind. Abhandlungen aus dem Mathematischen Seminar der Universität Hamburg, 36(1):9-15, 1971. doi:10.1007/BF02995904.

[36] C. Annavarapu, M. Hautefeuille, and J. E. Dolbow. A robust Nitsche's formulation for interface problems. Computer Methods in Applied Mechanics and Engineering, 225:44-54, 2012. doi:10.1016/j.cma.2012.03.008.

[37] F. de Prenter, C. V. Verhoosel, G. J. van Zwieten, and E. H. van Brummelen. Condition number analysis and preconditioning of the finite cell method. Computer Methods in Applied Mechanics and Engineering, 316:297-327, 2017. doi:10.1016/j.cma.2016.07.006.

[38] S. Badia, F. Verdugo, and A. F. Martín. The aggregated unfitted finite element method for elliptic problems. Computer Methods in Applied Mechanics and Engineering, 336:533-553, 2018. doi:10.1016/j.cma.2018.03.022.

[39] E. Burman and P. Zunino. Numerical approximation of large contrast problems with the unfitted Nitsche method. In Frontiers in Numerical Analysis-Durham 2010, pages 227-282. Springer, 2011. doi:10.1007/978-3-642-23914-4_4.

[40] F. Kummer. Extended discontinuous Galerkin methods for two-phase flows: the spatial discretization. International Journal for Numerical Methods in Engineering, 109(2):259-289, 2017. doi:10.1002/nme.5288.

[41] C. Lehrenfeld. High order unfitted finite element methods on level set domains using isoparametric mappings. Computer Methods in Applied Mechanics and Engineering, 300:716-733, 2016. doi:10.1016/j.cma.2015.12.005.

[42] J. Guzmán, M. A. Sánchez, and M. Sarkis. A finite element method for high-contrast interface problems with error estimates independent of contrast. Journal of Scientific Computing, 73(1):330-365, 2017. doi:10.1007/s10915-0170415-x.

[43] K. Li, N. M. Atallah, G. A. Main, and G. Scovazzi. The Shifted Interface Method: A flexible approach to embedded interface computations. International Journal for Numerical Methods in Engineering, 2019. doi:10.1002/nme.6231.

[44] C. Gürkan and A. Massing. A stabilized cut discontinuous Galerkin framework for elliptic boundary value and interface problems. Computer Methods in Applied Mechanics and Engineering, 348:466-499, 2019. doi:10.1016/j.cma.2018.12.041.

[45] R. Codina and S. Badia. On the design of discontinuous Galerkin methods for elliptic problems based on hybrid formulations. Computer Methods in Applied Mechanics and Engineering, 263:158-168, 2013. doi:10.1016/j.cma.2013.05.004.

[46] M. Ruess, D. Schillinger, A. I. Oezcan, and E. Rank. Weak coupling for isogeometric analysis of non-matching and trimmed multi-patch geometries. Computer Methods in Applied Mechanics and Engineering, 269:46-71, 2014. doi:10.1016/j.cma.2013.10.009.

[47] M. Elhaddad, N. Zander, T. Bog, L. Kudela, S. Kollmannsberger, J. Kirschke, T. Baum, M. Ruess, and E. Rank. Multi-level hp-finite cell method for embedded interface problems with application in biomechanics. International journal for numerical methods in biomedical engineering, 34(4):e2951, 2018. doi:10.1002/cnm.2951.

[48] C. Helzel, M. Berger, and R. Leveque. A high-resolution rotated grid method for conservation laws with embedded geometries. SIAM Journal on Scientific Computing, 26(3):785-809, 2005. doi:10.1137/S106482750343028X.

[49] P. Bastian and C. Engwer. An unfitted finite element method using discontinuous Galerkin. International journal for numerical methods in engineering, 79(12):1557-1576, 2009. doi:10.1002/nme.2631.

[50] E. Neiva, F. Verdugo, A. F. Martín, and S. Badia. The aggregated unfitted finite element method on parallel tree-based adaptive meshes. arXiv preprint arXiv:2006.05373v1, 2020. 
[51] S. Badia, A. F. Martín, and F. Verdugo. Mixed aggregated finite element methods for the unfitted discretization of the Stokes problem. SIAM Journal on Scientific Computing, 40(6):B1541-B1576, 2018. doi:10.1137/18M1185624.

[52] P. Huang, H. Wu, and Y. Xiao. An unfitted interface penalty finite element method for elliptic interface problems. Computer Methods in Applied Mechanics and Engineering, 323:439-460, 2017. doi:10.1016/j.cma.2017.06.004.

[53] E. Burman, M. Cicuttin, G. Delay, and A. Ern. An unfitted hybrid high-order method with cell agglomeration for elliptic interface problems. SIAM Journal on Scientific Computing, in press.

[54] F. Verdugo, A. F. Martín, and S. Badia. Distributed-memory parallelization of the aggregated unfitted finite element method. Computer Methods in Applied Mechanics and Engineering, 357:112583, 2019. doi:10.1016/j.cma.2019.112583.

[55] D. N. Arnold, F. Brezzi, B. Cockburn, and L. D. Marini. Unified analysis of discontinuous Galerkin methods for elliptic problems. SIAM journal on numerical analysis, 39(5):1749-1779, 2002. doi:10.1137/S0036142901384162.

[56] S. Badia, A. F. Martín, and J. Principe. FEMPAR: An Object-Oriented Parallel Finite Element Framework. Archives of Computational Methods in Engineering, 25(2):195-271, 2018. doi:10.1007/s11831-017-9244-1.

[57] C. Burstedde, L. C. Wilcox, and O. Ghattas. p4est: Scalable algorithms for parallel adaptive mesh refinement on forests of octrees. SIAM Journal on Scientific Computing, 33(3):1103-1133, 2011. doi:10.1137/100791634.

[58] O. Marco, R. Sevilla, Y. Zhang, J. J. Ródenas, and M. Tur. Exact 3D boundary representation in finite element analysis based on Cartesian grids independent of the geometry. International Journal for Numerical Methods in Engineering, 103(6):445-468, 2015. doi:10.1002/nme.4914.

[59] M. Olm, S. Badia, and A. F. Martín. On a general implementation of $h$ - and $p$-adaptive curl-conforming finite elements. Advances in Engineering Software, 132:74-91, 2019. doi:10.1016/j.advengsoft.2019.03.006.

[60] S. Badia, A. F. Martín, E. Neiva, and F. Verdugo. A generic finite element framework on parallel tree-based adaptive meshes. SIAM Journal on Scientific Computing, 42(6):C436-C468, 2020. doi:10.1137/20M1328786.

[61] A. Toselli and O. B. Widlund. Domain Decomposition Methods - Algorithms and Theory, volume 34 of Springer Series in Computational Mathematics. Springer Berlin Heidelberg, Berlin, Heidelberg, 2005. doi:10.1007/b137868.

[62] A. Ern and J.-L. Guermond. Theory and practice of finite elements, volume 159. Springer Science \& Business Media, 2013. doi:10.1007/978-1-4757-4355-5.

[63] R. Becker, E. Burman, and P. Hansbo. A Nitsche extended finite element method for incompressible elasticity with discontinuous modulus of elasticity. Computer Methods in Applied Mechanics and Engineering, 198(41-44): 3352-3360, 2009. doi:10.1016/j.cma.2009.06.017.

[64] E. Burman and P. Hansbo. Deriving robust unfitted finite element methods from augmented Lagrangian formulations. In Geometrically unfitted finite element methods and applications, pages 1-24. Springer, 2017. doi:10.1007/978-3319-71431-8_1.

[65] S. C. Brenner. Korn's inequalities for piecewise h1 vector fields. Mathematics of Computation, pages 1067-1087, 2004. doi:10.1090/S0025-5718-03-01579-5.

[66] Z. Chen and J. Zou. Finite element methods and their convergence for elliptic and parabolic interface problems. Numerische Mathematik, 79(2):175-202, 1998. doi:10.1007/s002110050336.

[67] L.-Y. Li, P. Bettess, J. Bull, T. Bond, and I. Applegarth. Theoretical formulations for adaptive finite element computations. Communications in Numerical Methods in Engineering, 11(10):857-868, 1995. doi:10.1002/cnm.1640111010.

[68] P. Díez and A. Huerta. A unified approach to remeshing strategies for finite element h-adaptivity. Computer Methods in Applied Mechanics and Engineering, 176(1-4):215-229, 1999. doi:10.1016/S0045-7825(98)00338-7.

[69] P. J. Roache. Code verification by the method of manufactured solutions. J. Fluids Eng., 124(1):4-10, 2002. doi:10.1115/1.1436090.

[70] N. Sukumar, D. L. Chopp, N. Moës, and T. Belytschko. Modeling holes and inclusions by level sets in the extended finite-element method. Computer Methods in Applied Mechanics and Engineering, 190(46-47):6183-6200, 2001. doi:10.1016/S0045-7825(01)00215-8.

[71] L. Demkowicz. Computing with hp-adaptive finite elements: Volume 1 one and two dimensional elliptic and maxwell problems. Chapman and Hall/CRC, 2006. doi:10.1201/9781420011685.

[72] GAMG online documentation. https://www.mcs.anl.gov/petsc/petsc-current/docs/manualpages/PC/ PCGAMG . html.

[73] S. Balay, S. Abhyankar, M. F. Adams, J. Brown, P. Brune, K. Buschelman, L. Dalcin, A. Dener, V. Eijkhout, W. D. Gropp, D. Kaushik, M. G. Knepley, D. A. May, L. C. McInnes, R. T. Mills, T. Munson, K. Rupp, P. Sanan, B. F. Smith, S. Zampini, H. Zhang, and H. Zhang. PETSc Users Manual. http: //www .mcs . anl . gov/petsc, 2019.

[74] Intel MKL PARDISO - Parallel Direct Sparse Solver Interface. https://software.intel.com/en-us/ articles/intel-mkl-pardiso.

[75] O. C. Zienkiewicz and J. Zhu. The superconvergent patch recovery (SPR) and adaptive finite element refinement. Computer Methods in Applied Mechanics and Engineering, 101(1-3):207-224, 1992. doi:10.1016/00457825(92)90023-D. 